\title{
Research Article \\ Heavy Scalar, Vector, and Axial-Vector Mesons in Hot and Dense Nuclear Medium
}

\begin{abstract}
Arvind Kumar
Department of Physics, Dr. B R Ambedkar National Institute of Technology Jalandhar, Jalandhar, Punjab 144011, India
\end{abstract}

Correspondence should be addressed to Arvind Kumar; iitd.arvind@gmail.com

Received 25 February 2014; Revised 28 May 2014; Accepted 18 June 2014; Published 22 July 2014

Academic Editor: Alexey A. Petrov

Copyright (C) 2014 Arvind Kumar. This is an open access article distributed under the Creative Commons Attribution License, which permits unrestricted use, distribution, and reproduction in any medium, provided the original work is properly cited. The publication of this article was funded by SCOAP $^{3}$.

\begin{abstract}
In this work we shall investigate the mass modifications of scalar mesons $\left(D_{0} ; B_{0}\right)$, vector mesons $\left(D^{*} ; B^{*}\right)$, and axial-vector mesons $\left(D_{1} ; B_{1}\right)$ at finite density and temperature of the nuclear medium. The above mesons are modified in the nuclear medium through the modification of quark and gluon condensates. We will find the medium modification of quark and gluon condensates within chiral SU(3) model through the medium modification of scalar-isoscalar fields $\sigma$ and $\zeta$ at finite density and temperature. These medium modified quark and gluon condensates will further be used through QCD sum rules for the evaluation of in-medium properties of the above mentioned scalar, vector, and axial vector mesons. We will also discuss the effects of density and temperature of the nuclear medium on the scattering lengths of the above scalar, vector, and axial-vector mesons. The study of the medium modifications of the above mesons may be helpful for understanding their production rates in heavy-ion collision experiments. The results of present investigations of medium modifications of scalar, vector, and axial-vector mesons at finite density and temperature can be verified in the compressed baryonic matter (CBM) experiment of FAIR facility at GSI, Germany.
\end{abstract}

\section{Introduction}

The motive behind the heavy-ion collision experiments at different experimental facilities is to explore the different phases of QCD phase diagram. These experiments help us to understand the nuclear matter properties for different values of temperatures and densities. The hadronic matter produced in heavy-ion collisions may undergo different phase transitions, for example, the liquid-gas phase transition, the kaon condensation, the restoration of chiral symmetry, and maybe the formation of quark gluon plasma [1-4]. The compressed baryonic matter (CBM) experiment of the FAIR project at GSI, Germany, may explore the phase of hadronic matter at high baryon densities and moderate temperatures. These kinds of phases may exist in the compact astrophysical objects, for example, neutron stars. The property of restoration of chiral symmetry is closely related to the medium modifications of hadrons [4]. The medium modifications of Kaons, $D$ mesons, and light vector mesons had been studied using different theoretical approaches, for example, chiral model [5-13], QCD sum rules [14-19], and coupled channel approach [20-22]. Due to interactions the properties of hadrons in the medium are found to be different as compared to their free space properties.

The medium modifications of heavy scalar, vector, and axial-vector mesons at finite density and temperature of the medium had been studied very rarely [23-26]. In the present investigation we will study the mass modifications of heavy scalar mesons $\left(D_{0}, B_{0}\right)$, vector mesons $\left(D^{*}, B^{*}\right)$, and axialvector mesons $\left(D_{1}, B_{1}\right)$ at finite densities and temperatures. The study of in-medium properties of scalar, vector, and axial-vector mesons will be helpful to understand their experimental production rates. The medium modification of charmed mesons may modify the experimental production of ground state charmonium $J / \psi$ and the excited charmonium states $\left(\psi^{\prime}\right.$ and $\left.\chi_{c}\right)$. The charmonium, $J / \psi$, may be produced due to the decay of the higher charmonium states. However, the vacuum threshold value of heavy meson pairs lies above the vacuum mass of the excited charmonium states. Now if these heavy mesons get modified (undergo mass drop in the medium) then the excited charmonium states may decay to the open charmed meson pairs instead of decaying 
to the ground state charmonium. Thus to understand the production of charmonium states in heavy-ion collisions it is very necessary to study the medium modification of the heavy scalar, vector, and axial-vector mesons. The medium modifications of heavy vector mesons may also help us in understanding the dilepton spectra produced in heavy-ion collision experiments [27-32]. The dileptons are considered as interesting probe to study the evolution of matter produced in heavy ion collision experiments as they do not undergo strong interactions in the medium. In [33] the production of open charm and charmonium in hot hadronic medium had been investigated using the statistical hadronization model at SPS/FAIR energies. In this work it was observed that the medium modifications of charmed hadrons do not lead to appreciable changes in cross-section for $D$ mesons production. This is because of large charm quark mass and different times scales for charm quark and charm hadron production. However, the charmonia yield is affected appreciably due to in-medium modifications.

The properties of scalar charm resonances $D_{s 0}(2317)$ and $D_{0}(2400)$ and hidden charm resonance, $X(3700)$, had been studied in [25] using coupled channel approach. In these studies the $D_{s 0}(2317)$ and $X(3700)$ were found to undergo a width of about 100 and $200 \mathrm{MeV}$, respectively, at nuclear matter density. However, for the $D_{0}(2400)$ mesons there was already large width of resonance in the free space and the medium effects were found to be weak as compared to $D_{s 0}(2317)$ and $X(3700)$. In [18] the mass splitting of $D-\bar{D}$ and $B-\bar{B}$ mesons had been studied using the QCD sum rules in the cold nuclear matter and the calculated values of mass splitting at nuclear saturation density were 60 and $130 \mathrm{MeV}$, respectively. The Borel transformed QCD sum rules had also been used to study the properties of pseudoscalar $D$ mesons [19] and vector mesons, $\rho, \omega$, and $\phi$ [17]. The properties of the scalar mesons $\left(D_{0}, B_{0}\right)$ in the cold nuclear matter using QCD sum rules have been investigated in [23]. The vectors mesons $\left(D^{*}, B^{*}\right)$ and axial-vector mesons $\left(D_{1}, B_{1}\right)$ had also been studied using QCD sum rules in cold nuclear matter in $[24]$. Note that in $[23,24]$ the properties of the meson were investigated at zero temperature and at normal nuclear matter density. However, in the present investigation we will find the in-medium masses of the scalar $\left(D_{0}, B_{0}\right)$ and vector $\left(D^{*}, B^{*}\right)$ and axial-vector $\left(D_{1}, B_{1}\right)$ mesons at finite temperatures as well as at the densities greater than the nuclear saturation density.

In the present work to investigate the properties of scalar, vector, and axial-vector mesons we will use the QCD sum rules and chiral SU(3) model [5]. Within QCD sum rules, the in-medium properties of mesons are related to the inmedium properties of quark and gluon condensates. We will investigate the in-medium properties of quark and gluon condensates using the chiral SU(3) model. Using chiral SU(3) model we will find the values of quark and gluon condensates at finite values of temperatures and baryonic densities. These values of condensates will further be used to find the medium modification of mesons using QCD sum rules. The chiral SU(3) model along with QCD sum rules had been used in the literature to investigate the in-medium modification of the charmonium states $J / \psi$ and $\eta_{c}$ [34].

The present paper is organized as follows. In Section 2 we will give a brief review of chiral SU(3) model. Then in Section 3 we will discuss how we will evaluate the inmedium modifications of the scalar, vector, and axial-vector mesons within QCD sum rules and using the properties of quark and gluon condensates as evaluated in the chiral SU(3) model. In Section 4 we will discuss the results of the present investigation and finally in Section 5 we will give a brief summary of present work.

\section{Chiral SU(3) Model}

In this section we will briefly review the chiral SU(3) model used in the present investigation for the in-medium properties of heavy mesons. The chiral SU(3) model is based on the broken scale invariance and nonlinear realization of chiral symmetry [35-40]. The model involves the Lagrangian densities describing, for example, kinetic energy terms, baryon-meson interactions, self-interactions of scalar mesons, vector mesons, symmetry breaking terms, and also the scale invariance breaking logarithmic potential terms.

For the investigation of hadron properties at finite temperature and densities we use the mean field approximation. Under this approximation all the meson fields are treated as classical fields and only the scalar and the vector fields contribute to the baryon-meson interactions. From the interaction Lagrangian densities, using the mean-field approximation, we derive the equations of motions for the scalar fields $\sigma$ and $\zeta$ and the dilaton field, $\chi$, in isospin symmetric nuclear medium. We solve these coupled equations to obtain the density and temperature dependence of scalar fields $\sigma$ and $\zeta$ and the dilaton field, $\chi$, in isospin symmetric nuclear medium [12]. The concept of broken scale invariance leading to the trace anomaly in (massless) QCD, $\theta_{\mu}^{\mu}=\left(\beta_{\mathrm{QCD}} / 2 g\right) G_{\mu \nu}^{a} G^{\mu \nu a}$, where $G_{\mu \nu}^{a}$ is the gluon field strength tensor of QCD, is simulated in the effective Lagrangian at tree level [41] through the introduction of the scale breaking terms [12]. Within chiral SU(3) model the scale breaking terms are written in terms of the dilaton field $\chi$ and also the scalar fields $\sigma$ and $\zeta$. From this we obtain the energy momentum tensor and this is compared with the energy momentum tensor of QCD which is written in terms of gluon condensates. In this way we extract the value of gluon condensates in terms of the scalar fields $\sigma$ and $\zeta$ and the dilaton field, $\chi$, and is given by the following equation [12]:

$$
\begin{aligned}
\left\langle\frac{\alpha_{s}}{\pi} G_{\mu \nu}^{a} G^{a \mu \nu}\right\rangle & \\
=\frac{8}{9} & {\left[(1-d) \chi^{4}+\left(\frac{\chi}{\chi_{0}}\right)^{2}\right.} \\
& \left.\times\left(m_{\pi}^{2} f_{\pi} \sigma+\left(\sqrt{2} m_{K}^{2} f_{K}-\frac{1}{\sqrt{2}} m_{\pi}^{2} f_{\pi}\right) \zeta\right)\right],
\end{aligned}
$$

where the value of parameter $d$ is 0.064 [10] and $m_{\pi}$ and $m_{K}$ denote the masses of pions and kaons and have values 
139 and $498 \mathrm{MeV}$, respectively. $f_{\pi}$ and $f_{K}$ are the decay constants having values 93.3 and $122 \mathrm{MeV}$, respectively. The symbols $\sigma, \zeta$, and $\chi$ denote the nonstrange scalar-isoscalar field, strange scalar-isoscalar field, and the dilaton field, respectively. $\chi_{0}$ denotes the value of the dilaton field in vacuum. The vacuum values of $\sigma, \zeta$, and $\chi$ are $-93.3,-106.6$, and $409.8 \mathrm{MeV}$, respectively. Note that in above equation the gluon condensate is written considering finite quark masses. If we have massless QCD, then only first term written in terms of dilaton field $\chi$ contributes to the gluon condensates. Using the above equation we obtain the values of scalar gluon condensates at different values of densities and temperatures of the nuclear medium.

\section{QCD Sum Rules for Scalar $\left(D_{0}, B_{0}\right)$, Vector $\left(D^{*}, B^{*}\right)$, and Axial-Vector $\left(D_{1}, B_{1}\right)$ Mesons}

In this section we will discuss the QCD sum rules [23, 24] which will be used later along with the chiral SU(3) model for the evaluation of in-medium properties of scalar, vector, and axial-vector mesons. To find the mass modification of the above discussed heavy mesons we will use the two-point correlation function $\Pi_{\mu \nu}(q)$,

$$
\Pi_{\mu \nu}(q)=i \int d^{4} x e^{i q \cdot x}\left\langle T\left\{J_{\mu}(x) J_{\nu}^{\dagger}(0)\right\}\right\rangle_{\rho_{B}, T^{*}}
$$

In the above equation $J_{\mu}(x)$ denotes the isospin averaged current, $x=x^{\mu}=\left(x^{0}, \mathbf{x}\right)$ is the four coordinate, $q=q^{\mu}=$ $\left(q^{0}, \mathbf{q}\right)$ is four momentum, and $T$ denotes the time ordered operation on the product of quantities in the brackets. From above definition it is clear that the two-point correlation function is actually a Fourier transform of the expectation value of the time ordered product of two currents. The twopoint correlation function for the scalar mesons is defined as

$$
\Pi(q)=i \int d^{4} x e^{i q \cdot x}\left\langle T\left\{J(x) J^{\dagger}(0)\right\}\right\rangle_{\rho_{B}, T} \cdot
$$

For the scalar, vector, and axial-vector mesons isospin average currents are given by the expressions

$$
\begin{gathered}
J(x)=J^{\dagger}(x)=\frac{\bar{c}(x) q(x)+\bar{q}(x) c(x)}{2}, \\
J_{\mu}(x)=J_{\mu}^{\dagger}(x)=\frac{\bar{c}(x) \gamma_{\mu} q(x)+\bar{q}(x) \gamma_{\mu} c(x)}{2} \\
J_{5 \mu}(x)=J_{5 \mu}^{\dagger}(x)=\frac{\bar{c}(x) \gamma_{\mu} \gamma_{5} q(x)+\bar{q}(x) \gamma_{\mu} \gamma_{5} c(x)}{2},
\end{gathered}
$$

respectively. Note that in above equations $q$ denotes the light $u$ or $d$ quark whereas $c$ denotes the heavy charm quark. Note that in the present work, instead of considering the mass splitting between particles and antiparticles, we emphasize on the mass shift of iso-doublet $D$ and $B$ mesons as a whole and, therefore, we consider the average in the definitions of scalar, vector, and iso-vector currents which is referred as centroid [19]. To find the mass splitting of particles and antiparticles in the nuclear medium one has to consider the even and odd part of QCD sum rules [18]. For example, in [18] the mass splitting between pseudoscalar $D$ and $\bar{D}$ mesons was investigated using the even and odd QCD sum rules whereas in $[19,23,24]$ the mass-shift of $D$ mesons was investigated under centroid approximation.

The two-point correlation function can be decomposed into the vacuum part, a static one-nucleon part, and pion bath contribution; that is, we can write

$$
\Pi_{\mu \nu}(q)=\Pi_{\mu \nu}^{0}(q)+\frac{\rho_{B}}{2 M_{N}} T_{\mu \nu}^{N}(q)+\Pi_{\mu \nu}^{P . B .}(q)
$$

where

$$
T_{\mu \nu}^{N}(\omega, \mathbf{q})=i \int d^{4} x e^{i q \cdot x}\left\langle N(p)\left|T\left\{J_{\mu}(x) J_{\nu}^{\dagger}(0)\right\}\right| N(p)\right\rangle .
$$

In the above equation $|N(p)\rangle$ denotes the isospin and spin averaged static nucleon state with the four-momentum $p=$ $\left(M_{N}, 0\right)$. The state is normalized as $\left\langle N(\mathbf{p}) \mid N\left(\mathbf{p}^{\prime}\right)\right\rangle=$ $(2 \pi)^{3} 2 p_{0} \delta^{3}\left(\mathbf{p}-\mathbf{p}^{\prime}\right)$. The third term, $\Pi_{\mu \nu}^{P . B .}(q)$ in (5) gives the contribution from pion bath at finite temperature. Note that in the present work instead of considering the contribution of pion bath the effects of finite temperature of the nuclear matter on the properties of $D$ and $B$ mesons will be evaluated through the temperature dependence of scalar fields $\sigma, \zeta$, and $\chi$. The temperature dependence of scalar fields $\sigma, \zeta$, and $\chi$ modify the nucleon properties in the medium and these modified nucleons further modify the in-medium properties of $D$ and $B$ mesons at finite temperature and density. In literature the properties of kaons and antikaons, $D$ mesons and charmonium had been studied at finite temperature of the nuclear matter using the above mentioned scalar fields $\sigma$, $\zeta$, and $\chi[7,8,12,13,34]$.

As discussed in [24], in the limit of the 3-vector $\mathbf{q} \rightarrow \mathbf{0}$, the correlation functions $T_{N}(\omega, \mathbf{q})$ can be related to the $D^{*} N$ and $D_{1} N$ scattering $T$-matrices. Thus we write [24]

$$
\begin{aligned}
& \mathscr{T}_{D^{*} N}\left(M_{D^{*}}, 0\right)=8 \pi\left(M_{N}+M_{D^{*}}\right) a_{D^{*}} \\
& \mathscr{T}_{D_{1} N}\left(M_{D_{1}}, 0\right)=8 \pi\left(M_{N}+M_{D_{1}}\right) a_{D_{1}} .
\end{aligned}
$$

In above equation $a_{D^{*}}$ and $a_{D_{1}}$ are the scattering lengths of $D^{*} N$ and $D_{1} N$, respectively. Similarly, we can also write the scattering $T$ matrix corresponding to $D_{0} N\left(D_{0}\right.$ is a scalar meson) in terms of the scattering lengths [23],

$$
\mathscr{T}_{D_{0} N}\left(M_{D_{0}}, 0\right)=8 \pi\left(M_{N}+M_{D_{0}}\right) a_{D_{0}} .
$$

Near the pole positions of the scalar, vector, and axialvector mesons the phenomenological spectral densities can 
be parameterized with three unknown parameters $a, b$, and $c$; that is, we write $[19,23,24]$

$$
\begin{aligned}
& \rho(\omega, 0) \\
& \left.=-\frac{f_{D_{0} / D^{*} / D_{1}}^{2} M_{D_{0} / D^{*} / D_{1}}^{2} \operatorname{Im}\left[\frac{\mathscr{T}_{D_{0} / D^{*} / D_{1} N}(\omega, \mathbf{0})}{\pi}\right]}{\left(\omega^{2}-M_{D_{0} / D^{*} / D_{1}}^{2}+i \varepsilon\right)^{2}}\right] \\
& \quad+\cdots=a \frac{d}{d \omega^{2}} \delta^{\prime}\left(\omega^{2}-M_{D_{0} / D^{*} / D_{1}}^{2}\right) \\
& \quad+b \delta\left(\omega^{2}-M_{D_{0} / D^{*} / D_{1}}^{2}\right)+c \theta\left(\omega^{2}-s_{0}\right) .
\end{aligned}
$$

The term denoted by $\cdots$ represents the continuum contributions. The first term denotes the double-pole term and corresponds to the on-shell effects of the $T$-matrices,

$$
a=-8 \pi\left(M_{N}+M_{D_{0} / D^{*} / D_{1}}\right) a_{D_{0} / D^{*} / D_{1}} f_{D_{0} / D^{*} / D_{1}}^{2} M_{D_{0} / D^{*} / D_{1}}^{2} .
$$

Now we will write the relation between the scattering length of mesons and their in-medium mass-shift. For this first we note that the shift of squared mass of mesons can be written in terms of the parameter $a$ appearing in (9) through relation [17],

$$
\begin{aligned}
\Delta m_{D_{0} / D^{*} / D_{1}}^{2} & =\frac{\rho_{N}}{2 M_{N}} \frac{a}{f_{D_{0} / D^{*} / D_{1}}^{2} M_{D_{0} / D^{*} / D_{1}}^{2}} \\
& =\frac{\rho_{N}}{2 M_{N}} 8 \pi\left(M_{N}+M_{D_{0} / D^{*} / D_{1}}\right) a_{D_{0} / D^{*} / D_{1}},
\end{aligned}
$$

where in the last term we used (10). The mass shift is now defined by the relation

$$
\delta M_{D_{0} / D^{*} / D_{1}}=\sqrt{m_{D_{0} / D^{*} / D_{1}}^{2}+\Delta m_{D_{0} / D^{*} / D_{1}}^{2}}-m_{D_{0} / D^{*} / D_{1}} .
$$

The second term in (9) denotes the single-pole term and corresponds to the off-shell (i.e., $\omega^{2} \neq M_{D_{0} / D^{*} / D_{1}}^{2}$ ) effects of the $T$-matrices. The third term denotes the continuum term or the remaining effects, where $s_{0}$ is the continuum threshold. The continuum threshold parameter $s_{0}$ define the scale below which the continuum contribution vanishes [42].

It can be observed from (11) and (12) that if we want to find the value of mass shift of mesons then we first need to find the value of unknown parameter $a$. For this we proceed as follows: we note that in the low energy limit, $\omega \rightarrow 0$, the $T_{N}(\omega, \mathbf{0})$ is equivalent to the Born term $T_{D_{0} / D^{*} / D_{1} N}^{B o r n}(\omega, \mathbf{0})$. We take into account the Born term at the phenomenological side,

$$
\begin{aligned}
T_{N}\left(\omega^{2}\right)= & T_{D_{0} / D^{*} / D_{1} N}^{\text {Born }}\left(\omega^{2}\right)+\frac{a}{\left(M_{D_{0} / D^{*} / D_{1}}^{2}-\omega^{2}\right)^{2}} \\
& +\frac{b}{M_{D_{0} / D^{*} / D_{1}}^{2}-\omega^{2}}+\frac{c}{s_{0}-\omega^{2}},
\end{aligned}
$$

with the constraint

$$
\frac{a}{M_{D_{0} / D^{*} / D_{1}}^{4}}+\frac{b}{M_{D_{0} / D^{*} / D_{1}}^{2}}+\frac{c}{s_{0}}=0 .
$$

Note that in (13) the phenomenological side of scattering amplitude for $q_{\mu} \neq 0$ is not exactly equal to Born term but there are contributions from other terms. However, for $\omega \rightarrow$ $0, T_{N}$ on left should be equal to $T^{\text {Born }}$ on right side of (13) and this requirement results in constraint given in (14). As we will discuss below the constraint (14) help in eliminating the parameter $c$ and scattering amplitude will be function of parameters $a$ and $b$ only. The Born terms to be used in (13) for scalar, vector, and axial-vector mesons are given by following relations $[23,24]$ :

$$
\begin{aligned}
& T_{D_{0} N}^{\text {Born }}(\omega, \boldsymbol{0})=\frac{2 f_{D_{0}}^{2} M_{D_{0}}^{2} M_{N}\left(M_{H}-M_{N}\right) g_{D_{0} N H}^{2}}{\left[\omega^{2}-\left(M_{H}-M_{N}\right)^{2}\right]\left[\omega^{2}-M_{D_{0}}^{2}\right]^{2}}, \\
& T_{D^{*} N}^{\text {Born }}(\omega, \mathbf{0})=\frac{2 f_{D^{*}}^{2} M_{D^{*}}^{2} M_{N}\left(M_{H}+M_{N}\right) g_{D^{*} N H}^{2}}{\left[\omega^{2}-\left(M_{H}+M_{N}\right)^{2}\right]\left[\omega^{2}-M_{D^{*}}^{2}\right]^{2}}, \\
& T_{D_{1} N}^{\text {Born }}(\omega, \mathbf{0})=\frac{2 f_{D_{1}}^{2} M_{D_{1}}^{2} M_{N}\left(M_{H}-M_{N}\right) g_{D_{1} N H}^{2}}{\left[\omega^{2}-\left(M_{H}-M_{N}\right)^{2}\right]\left[\omega^{2}-M_{D_{1}}^{2}\right]^{2}} .
\end{aligned}
$$

In the above equations $g_{D_{0} N H}, g_{D^{*} N H}$, and $g_{D_{1} N H}$ are the coupling constants. $M_{H}$ is the mass of the hadron; for example, corresponding to charm mesons we have $\Lambda_{c}$ and $\Sigma_{c}$, whereas corresponding to bottom mesons we have the hadrons $\Lambda_{b}$ and $\Sigma_{b}$. Corresponding to charm mesons we take the average value of the masses of $M_{\Lambda}$ and $M_{\Sigma_{c}}$ and it is equal to $2.4 \mathrm{GeV}$. For the case of mesons having bottom quark, $b$, we consider the average value of masses of $\Lambda_{b}$ and $\Sigma_{b}$ and it is equal to $5.7 \mathrm{GeV}$.

Now we write the equation for the Borel transformation of the scattering matrix on the phenomenological side and equate that to the Borel transformation of the scattering matrix for the operator expansion side. For the scalar meson, $D_{0}$, the Borel transformation equation is written as [23]

$$
\begin{aligned}
& a\left\{\frac{1}{M^{2}} e^{-M_{D_{0}}^{2} / M^{2}}-\frac{s_{0}}{M_{D_{0}}^{4}} e^{-s_{0} / M^{2}}\right\} \\
& \quad+b\left\{e^{-M_{D_{0}}^{2} / M^{2}}-\frac{s_{0}}{M_{D_{0}}^{2}} e^{-s_{0} / M^{2}}\right\} \\
& +A\left[\frac{1}{\left(M_{H}-M_{N}\right)^{2}-M_{D_{0}}^{2}}-\frac{1}{M^{2}}\right] e^{-M_{D_{0}}^{2} / M^{2}} \\
& \quad-\frac{A}{\left(M_{H}-M_{N}\right)^{2}-M_{D_{0}}^{2}} e^{-\left(M_{H}-M_{N}\right)^{2} / M^{2}} \\
& =\left\{\frac{m_{c}\langle\bar{q} q\rangle_{N}}{2}-\left\langle q^{\dagger} i D_{0} q\right\rangle_{N}+\frac{m_{c}^{2}\left\langle q^{\dagger} i D_{0} q\right\rangle_{N}}{M^{2}}\right\} e^{-m_{c}^{2} / M^{2}}
\end{aligned}
$$




$$
\begin{aligned}
& -\left\{\frac{2 m_{c}\left\langle\bar{q} i D_{0} i D_{0} q\right\rangle_{N}}{M^{2}}-\frac{m_{c}^{3}\left\langle\bar{q} i D_{0} i D_{0} q\right\rangle_{N}}{M^{4}}\right\} e^{-m_{c}^{2} / M^{2}} \\
& +\frac{1}{16}\left\langle\frac{\alpha_{s} G G}{\pi}\right\rangle_{N} \int_{0}^{1} d x\left(1+\frac{\widetilde{m}_{c}^{2}}{M^{2}}\right) e^{-\widetilde{m}_{c}^{2} / M^{2}} \\
& -\frac{1}{48 M^{4}}\left\langle\frac{\alpha_{s} G G}{\pi}\right\rangle_{N} \int_{0}^{1} d x \frac{1-x}{x} \widetilde{m}_{c}^{4} e^{-\widetilde{m}_{c}^{2} / M^{2}}
\end{aligned}
$$

where $A=2 f_{D_{0}}^{2} M_{D_{0}}^{2} M_{N}\left(M_{H}-M_{N}\right) g_{D_{0} N H}^{2} /\left(\left(M_{H}-M_{N}\right)^{2}-\right.$ $\left.M_{D_{0}}^{2}\right)$.

Note that in (16) we have two unknown parameters $a$ and $b$. We differentiate (16) w.r.t. $1 / M^{2}$ so that we could have two equations and two unknowns. By solving those two coupled equations we will be able to get the values of parameters $a$ and $b$. Same procedure will be applied to obtain the values of parameters $a$ and $b$ corresponding to vector and axial-vector mesons. For vector meson, $D^{*}$, the Borel transformation equation is given by [24]

$$
\begin{aligned}
a\left\{\frac{1}{M^{2}} e^{-M_{D^{*}}^{2} / M^{2}}-\frac{s_{0}}{M_{D^{*}}^{4}} e^{-s_{0} / M^{2}}\right\} & +b\left\{e^{-M_{D^{*}}^{2} / M^{2}}-\frac{s_{0}}{M_{D^{*}}^{2}} e^{-s_{0} / M^{2}}\right\} \\
& +B\left[\frac{1}{\left(M_{H}+M_{N}\right)^{2}-M_{D^{*}}^{2}}-\frac{1}{M^{2}}\right] e^{-M_{D^{*}}^{2} / M^{2}} \\
& -\frac{B}{\left(M_{H}+M_{N}\right)^{2}-M_{D^{*}}^{2}} e^{-\left(M_{H}+M_{N}\right)^{2} / M^{2}} \\
= & \left\{-\frac{m_{c}\langle\bar{q} q\rangle_{N}}{2}-\frac{2\left\langle q^{\dagger} i D_{0} q\right\rangle_{N}}{3}+\frac{m_{c}^{2}\left\langle q^{\dagger} i D_{0} q\right\rangle_{N}}{M^{2}}\right\} \\
& \times e^{-m_{c}^{2} / M^{2}}+\frac{m_{c}\left\langle\bar{q} g_{s} \sigma G q\right\rangle_{N}}{3 M^{2}} e^{-m_{c}^{2} / M^{2}} \\
& +\left\{\frac{8 m_{c}\left\langle\bar{q} i D_{0} i D_{0} q\right\rangle_{N}}{3 M^{2}}-\frac{m_{c}^{3}\left\langle\bar{q}^{i} D_{0} i D_{0} q\right\rangle_{N}}{M^{4}}\right\} e^{-m_{c}^{2} / M^{2}} \\
& +\frac{1}{24}\left\langle\frac{\alpha_{s} G G}{\pi}\right\rangle_{N} \int_{0}^{1} d x\left(1+\frac{\widetilde{m}_{c}^{2}}{2 M^{2}}\right) e^{-\widetilde{m}_{c}^{2} / M^{2}} \\
\pi & \left.\frac{1}{\pi}\right\rangle_{N} \int_{0}^{1} d x \frac{1-x}{x}\left(\widetilde{m}_{c}^{2}-\frac{\tilde{m}_{c}^{4}}{M^{2}}\right) e^{-\widetilde{m}_{c}^{2} / M^{2}},
\end{aligned}
$$

where $B=2 f_{D^{*}}^{2} M_{D^{*}}^{2} M_{N}\left(M_{H}+M_{N}\right) g_{D^{*} N H}^{2} /\left(\left(M_{H}+M_{N}\right)^{2}-\right.$ $\left.M_{D^{*}}^{2}\right)$. For the axial-vector mesons, $D_{1}$, the Borel transformation equation is given by [24]

$$
\begin{aligned}
& a\left\{\frac{1}{M^{2}} e^{-M_{D_{1}}^{2} / M^{2}}-\frac{s_{0}}{M_{D_{1}}^{4}} e^{-s_{0} / M^{2}}\right\} \\
& +b\left\{e^{-M_{D_{1}}^{2} / M^{2}}-\frac{s_{0}}{M_{D_{1}}^{2}} e^{-s_{0} / M^{2}}\right\}
\end{aligned}
$$

$$
\begin{aligned}
& +C\left[\frac{1}{\left(M_{H}-M_{N}\right)^{2}-M_{D_{1}}^{2}}-\frac{1}{M^{2}}\right] e^{-M_{D_{1}}^{2} / M^{2}} \\
& -\frac{C}{\left(M_{H}-M_{N}\right)^{2}-M_{D_{1}}^{2}} e^{-\left(M_{H}-M_{N}\right)^{2} / M^{2}} \\
= & \left.\left\{\frac{m_{c}\langle\bar{q} q\rangle_{N}}{2}-\frac{2\left\langle q^{\dagger} i D_{0} q\right\rangle_{N}}{3}+\frac{m_{c}^{2}\left\langle q^{\dagger} i D_{0} q\right\rangle_{N}}{M^{2}}\right\} e^{-m_{c}^{2} / M^{2}}\right\} \\
& -\frac{m_{c}\left\langle\bar{q} g_{s} \sigma G q\right\rangle_{N}}{3 M^{2}} e^{-m_{c}^{2} / M^{2}} \\
& -\left\{\frac{8 m_{c}\left\langle\bar{q} i D_{0} i D_{0} q\right\rangle_{N}}{3 M^{2}}-\frac{m_{c}^{3}\left\langle\bar{q}^{i} D_{0} i D_{0} q\right\rangle_{N}}{M^{4}}\right\} e^{-m_{c}^{2} / M^{2}} \\
& -\frac{1}{24}\left\langle\frac{\alpha_{s} G G}{\pi}\right\rangle_{N} \int_{0}^{1} d x\left(1+\frac{\widetilde{m}_{c}^{2}}{2 M^{2}}\right) e^{-\widetilde{m}_{c}^{2} / M^{2}} \\
& +\frac{1}{48 M^{2}}\left\langle\frac{\alpha_{s} G G}{\pi}\right\rangle_{N} \int_{0}^{1} d x \frac{1-x}{x}\left(\widetilde{m}_{c}^{2}-\frac{\widetilde{m}_{c}^{4}}{M^{2}}\right) e^{-\widetilde{m}_{c}^{2} / M^{2}}
\end{aligned}
$$

where $C=2 f_{D_{1}}^{2} M_{D_{1}}^{2} M_{N}\left(M_{H}-M_{N}\right) g_{D_{1} N H}^{2} /\left(\left(M_{H}-M_{N}\right)^{2}-\right.$ $M_{D_{1}}^{2}$ ). In the above equations $\widetilde{m}_{c}^{2}=m_{c}^{2} / x$.

As discussed earlier, in determining the properties of hadrons from QCD sum rules, we will use the values of quark and gluon condensates as calculated using chiral SU(3) model. Any operator $\mathcal{O}$ on OPE side can be written as $[17,42$, 43]

$$
\begin{aligned}
\mathcal{O}_{\rho_{B}}= & \mathcal{O}_{\text {vacuum }}+4 \int \frac{d^{3} p}{(2 \pi)^{3} 2 E_{p}} n_{F}\langle N(p)|\mathcal{O}| N(p)\rangle \\
& +3 \int \frac{d^{3} k}{(2 \pi)^{3} 2 E_{k}} n_{B}\langle\pi(k)|\mathcal{O}| \pi(k)\rangle \\
= & \mathcal{O}_{\text {vacuum }}+\frac{\rho_{B}}{2 M_{N}} \mathcal{O}_{N}+\mathcal{O}_{P . B .} .
\end{aligned}
$$

In the above equation, $\mathcal{O}_{\rho_{B}}$ gives us the expectation value of the operator at finite baryonic density. The term $\mathcal{O}_{\text {vacuum }}$ stands for the vacuum expectation value of the operator, $\mathcal{O}_{N}$ gives us the nucleon expectation value of the operator, and $\mathcal{O}_{P . B}$. denotes the contribution from the pion bath at finite temperature. Consider $n_{B}=\left[e^{E_{k} / T}-1\right]^{-1}$ and $n_{F}=$ $\left[e^{\left(E_{p}-\mu_{N}\right) / T}-1\right]^{-1}$ are the thermal Boson and Fermion distribution functions. Within the chiral SU(3) model the quark and gluon condensates can be expressed in terms of scalar fields $\sigma, \zeta$, and $\chi$. As discussed earlier, the finite temperature effects in the present investigation will be evaluated through the scalar fields and, therefore, contribution of third term will not be considered. However, for completeness we will compare the temperature dependence of scalar quark and scalar gluon condensates at zero baryon density as evaluated in the present work with the situation when the temperature dependence is evaluated using only pion bath contribution 
[42]. Thus within chiral SU(3) model, we can find the values of $\mathcal{O}_{\rho_{B}}$ at finite density of the nuclear medium and hence can find $\mathscr{O}_{N}$ using

$$
\mathcal{O}_{N}=\left[\mathcal{O}_{\rho_{B}}-\mathcal{O}_{\text {vacuum }}\right] \frac{2 M_{N}}{\rho_{B}} .
$$

The quark condensate, $\bar{q} q$, can be extracted from the explicit symmetry breaking term of the Lagrangian density and is given by

$$
\begin{aligned}
\sum_{i} m_{i} \overline{q_{i}} q_{i}= & -\mathscr{L}_{S B}=\left(\frac{\chi}{\chi_{0}}\right)^{2} \\
& \times\left[m_{\pi}^{2} f_{\pi} \sigma+\left(\sqrt{2} m_{k}^{2} f_{k}-\frac{1}{\sqrt{2}} m_{\pi}^{2} f_{\pi}\right) \zeta\right] .
\end{aligned}
$$

In our present investigation of hadron properties, we are interested in light quark condensates, $\bar{u} u$ and $\bar{d} d$, which are proportional to the nonstrange scalar field $\sigma$ within chiral $\mathrm{SU}(3)$ model. Considering equal mass of light quarks, $u$ and $d$ that is., $m_{u}=m_{d}=m_{q}=0.006 \mathrm{GeV}$, we can write,

$$
\langle\bar{q} q\rangle_{\rho_{B}}=\frac{1}{2 m_{q}}\left(\frac{\chi}{\chi_{0}}\right)^{2}\left[m_{\pi}^{2} f_{\pi} \sigma\right] .
$$

The condensate $\left\langle\bar{q} g_{s} \sigma G q\right\rangle_{\rho_{B}}$ is given by the following [44]:

$$
\left\langle\bar{q} g_{s} \sigma G q\right\rangle_{\rho_{B}}=\lambda^{2}\langle\bar{q} q\rangle_{\rho_{B}}+3.0 \mathrm{GeV}^{2} \rho_{B}
$$

Also we write [44]

$$
\left\langle\bar{q} i D_{0} i D_{0} q\right\rangle_{\rho_{B}}+\frac{1}{8}\left\langle\bar{q} g_{s} \sigma G q\right\rangle_{\rho_{B}}=0.3 \mathrm{GeV}^{2} \rho_{B} .
$$

As discussed above the quark condensate, $\langle\bar{q} q\rangle_{\rho_{B}}$, can be calculated within the chiral SU(3) model. This value of $\langle\bar{q} q\rangle_{\rho_{B}}$ can be used through (23) and (24) to calculate the value of condensates $\left\langle\bar{q} g_{s} \sigma G q\right\rangle_{\rho_{B}}$ and $\left\langle\bar{q} i D_{0} i D_{0} q\right\rangle_{\rho_{B}}$ within chiral $\mathrm{SU}(3)$ model. The value of condensate $\left\langle q^{\dagger} i D_{0} q\right\rangle$ is equal to $0.18 \mathrm{GeV}^{2} \rho_{B}[44]$.

At finite temperature and zero baryon density we can write the expectation values of quark condensates and scalar gluon condensates as [42]

$$
\begin{aligned}
\langle\bar{q} q\rangle_{T}=\langle\bar{q} q\rangle_{\text {vacuum }} & {\left[1-\frac{T^{2}}{8 f_{\pi}^{2}} B_{1}\left(\frac{m_{\pi}}{T}\right)\right], } \\
\left\langle\frac{\alpha_{s}}{\pi} G_{\mu \nu}^{a} G^{a \mu \nu}\right\rangle_{T}= & \left\langle\frac{\alpha_{s}}{\pi} G_{\mu \nu}^{a} G^{a \mu \nu}\right\rangle_{\text {vacuum }} \\
& -\frac{1}{9} m_{\pi}^{2} T^{2} B_{1}\left(\frac{m_{\pi}}{T}\right),
\end{aligned}
$$

respectively. In (25) and (26), $B_{1}(x)$ $\left(6 / \pi^{2}\right) \int_{x}^{\infty} d y \sqrt{y^{2}-x^{2}} /\left(e^{y}-1\right)$. From (25) and (26) we observe that the contribution from pion bath to the expectation values of operator arises only at finite temperature. Note that in (16), (17), and (18) we need the nucleon expectation values of various condensates which can be evaluated in general using (20). In (1), (22), (23), and (24) the values of condensates are given at finite value of baryonic density. To find the corresponding nucleon expectation values of various condensates we use the values of condensates at finite baryonic density from (1), (22), (23), and (24) in (20). The equation (20) is then further used in (16), (17), and (18) for calculation of medium modifications of $D$ mesons.

It may be noted that the QCD sum rules for the evaluation of in-medium properties of scalar mesons, $B_{0}$, vector mesons, $B^{*}$, and axial-vector mesons, $B_{1}$, can be written by replacing masses of charmed mesons, $D_{0}, D^{*}$, and $D_{1}$, by corresponding masses of bottom mesons $B_{0}, B^{*}$, and $B_{1}$ in (16), (17), and (18), respectively. Also the bare charm quark mass, $m_{c}$, will be replaced by the mass of bottom quark, $m_{b}$.

\section{Results and Discussions}

In this section we will present the results of our investigation of in-medium properties of scalar $\left(D_{0}, B_{0}\right)$, vector $\left(D^{*}, B^{*}\right)$, and axial-vector mesons $\left(D_{1}, B_{1}\right)$. The nuclear matter saturation density used in the present investigation is $0.15 \mathrm{fm}^{-3}$. The values of various coupling constants $g_{D_{0} N \Lambda_{c}} \approx g_{D_{0} N \Sigma_{c}} \approx$ $g_{B_{0} N \Lambda_{b}} \approx g_{B_{0} N \Sigma_{b}}$ are approximated to 6.74 [23]. The coupling constants $g_{D^{*} N \Lambda_{c}} \approx g_{D^{*} N \Sigma_{c}} \approx g_{D_{1} N \Lambda_{c}} \approx g_{D_{1} N \Sigma_{c}} \approx g_{B^{*} N \Lambda_{b}} \approx$ $g_{B^{*} N \Sigma_{b}} \approx g_{B_{1} N \Lambda_{b}} \approx g_{B_{1} N \Sigma_{b}}$ are approximated to 3.86 [24]. The masses of mesons $M_{D_{0}}, M_{B_{0}}, M_{D^{*}}, M_{B^{*}}, M_{D_{1}}$, and $M_{B_{1}}$ to be used in present investigation are 2.355, 5.74, 2.01, 5.325, 2.42 , and $5.75 \mathrm{GeV}$, respectively. The values of decay constants $f_{D_{0}}, f_{B_{0}}, f_{D^{*}}, f_{B^{*}}, f_{D_{1}}$, and $f_{B_{1}}$ are $0.334,0.28,0.270,0.195$, 0.305 , and $0.255 \mathrm{GeV}$, respectively. The values of threshold parameters, $s^{0}$, corresponding to $D_{0}, B_{0}, D^{*}, B^{*}, D_{1}$, and $B_{1}$ mesons are $8,39,6.5,35,8.5$, and $39 \mathrm{GeV}^{2}$, respectively [23, 24]. As discussed earlier the mass-shift of scalar, vector, and axial-vector mesons is calculated through the parameter $a$ which is related to scattering length through (10) to (12). This parameter $a$, for example, for $D^{0}$, is calculated by solving the coupled equations as discussed after (16) and is subjected to the medium modifications through the medium dependence of condensates. The medium dependence of condensates is further evaluated through the scalar fields $\sigma, \zeta$, and $\chi$. The various coupling constants listed above, the decay constants of $D$ and $B$ mesons, and the threshold parameter $s_{0}$ are not subjected to the medium modifications. In the present work we will show the variation of mass shift as a function of squared Borel mass parameter, $M$. The Borel window is chosen such that there is almost no change in the mass of $D$ and $B$ mesons w.r.t variation in Borel mass parameter. For the charmed scalar, $D_{0}$, vector, $D^{*}$, and axial-vector, $D_{1}$ mesons Borel windows are found to be (6.1-7.4), (4.5-5.4), and (6.57.6) $\mathrm{GeV}^{2}$, respectively. The Borel windows for bottom scalar, $B_{0}$, vector, $B^{*}$, and axial-vector, $B_{1}$ mesons are (33-39), (22$24)$, and (34-37) $\mathrm{GeV}^{2}$, respectively.

In the present work we are studying the in-medium masses of scalar, vector, and axial-vector mesons using QCD sum rules and chiral SU(3) model. Since we are evaluating the 


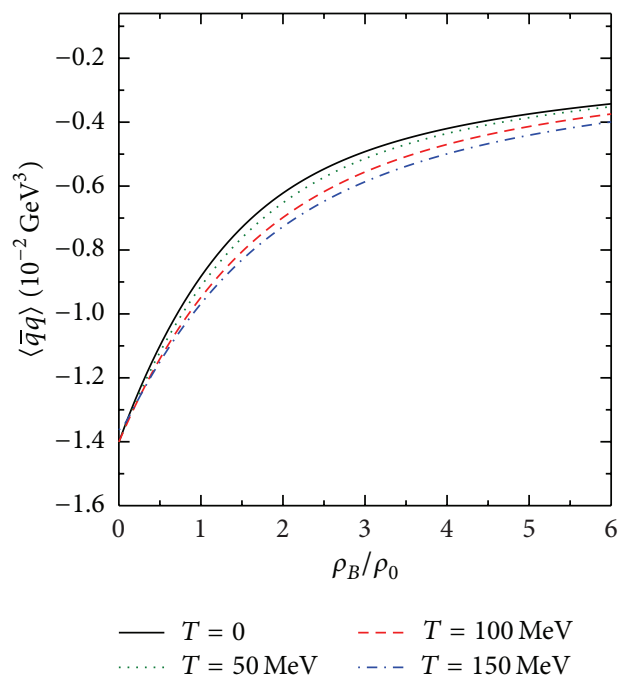

Figure 1: (Color online) The light quark condensate $\bar{q} q$ plotted as a function of density of the nuclear medium, in units of nuclear saturation density, for different values of temperatures $(T=$ $0,50,100$, and $150 \mathrm{MeV}$ ).

quark and gluon condensates within the chiral SU(3) model through the modification of scalar fields $\sigma$ and $\zeta$ and the scalar dilaton field $\chi$, so first we will discuss in short the effect of temperature and density of the medium on the values of scalar fields $\sigma$ and $\zeta$ and the scalar dilaton field $\chi$. We observe that as a function of density of the nuclear medium the magnitude of scalar fields decreases. For example, at nuclear saturation density, $\rho_{B}=\rho_{0}$, the drop in magnitude of the scalar fields $\sigma$ and $\zeta$ and the dilaton field $\chi$ is observed to be 33.49, 9.54 and $3.39 \mathrm{MeV}$, respectively. At baryon densities, $4 \rho_{0}$, these values changes to $63.41,14.51$ and $13.06 \mathrm{MeV}$, respectively.

At zero baryon density, we observe that the magnitude of the scalar fields $\sigma$ and $\zeta$ and the dilaton field $\chi$ decreases with the increase in the temperature. However, the change in the values of scalar fields with temperature of the medium is observed to be very small. The reason for the nonzero values of scalar fields at finite temperature and zero baryon density of the medium is the formation of baryon-antibaryon pairs $[36,45,46]$. At finite baryon densities, the magnitude of the scalar fields increases with increase in the temperature of symmetric nuclear medium. This also leads to the increase in the masses of the nucleons with the temperature of the nuclear medium for finite baryon densities [12]. At nuclear saturation density, $\rho_{0}$, the magnitude of the scalar fields, $\sigma$ and $\zeta$, and the dilaton field $\chi$ increases by 5.47,1.28 and $0.96 \mathrm{MeV}$, respectively, as we move from $T=0$ to $T=150 \mathrm{MeV}$, respectively.

In Figures 1 and 2 we show the variation of the light scalar quark condensate, $\bar{q} q$, given by (22) and the scalar gluon condensate, $G_{0}=\left\langle\left(\alpha_{s} / \pi\right) G_{\mu \nu}^{a} G^{a \mu \nu}\right\rangle$, given by (1), respectively, as a function of density of the symmetric nuclear medium. We show the results for temperatures, $T=0,50,100$, and $150 \mathrm{MeV}$, respectively. From (22) we observe that the value of the scalar quark condensate $\bar{q} q$ is directly proportional to the scalar-isoscalar field, $\sigma$. Therefore, the behavior of the

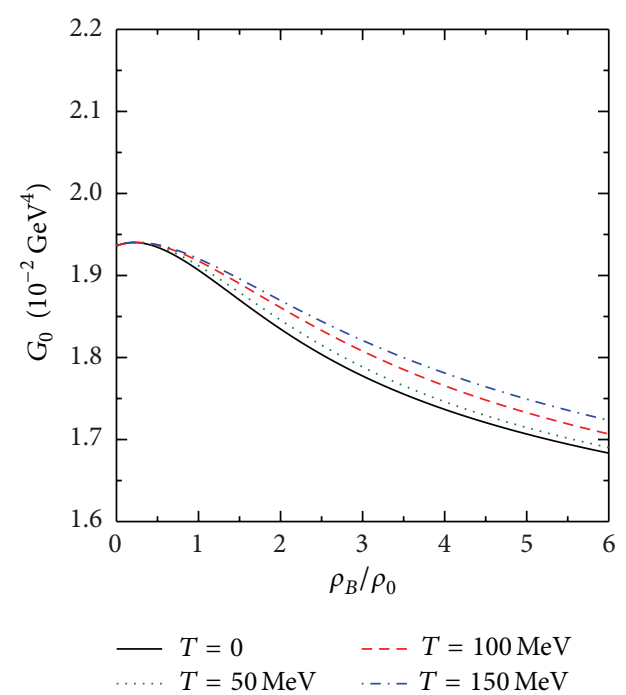

Figure 2: (Color online) The scalar gluon condensate $G_{0}$ plotted as a function of density of the nuclear medium, in units of nuclear saturation density, for different values of temperatures $(T=$ $0,50,100$, and $150 \mathrm{MeV})$.

$\bar{q} q$ as a function of temperature and density of the nuclear medium will be the same as that of $\sigma$ field. For given value of temperature of the nuclear medium the magnitude of the light quark condensate decreases with the increase in the density. For example, at $T=0$, the values of light quark condensate are observed to be $-0.8829 \times 10^{-2}$ and $-0.4200 \times 10^{-2} \mathrm{GeV}^{3}$ at baryon densities, $\rho_{B}=\rho_{0}$ and $4 \rho_{0}$, respectively. At temperature, $T=150 \mathrm{MeV}$, these values of quark condensates changes to $-0.9681 \times 10^{-2}$ and $-0.4993 \times 10^{-2} \mathrm{GeV}^{3}$, respectively. At zero baryon density the magnitude of the $\bar{q} q$ decreases with the increase in the temperature of the nuclear medium. At $\rho_{B}=0$, the values of $\bar{q} q$ are observed to be $-1.4014 \times 10^{-2}$, $-1.4014 \times 10^{-2},-1.4006 \times 10^{-2}$, and $-1.3628 \times 10^{-2} \mathrm{GeV}^{3}$ at temperatures $T=0,50,100$, and $150 \mathrm{MeV}$, respectively.

From Figure 2, we observe that the values of the scalar gluon condensates decrease with the increase in the density of the nuclear medium. At baryon density, $\rho_{B}=\rho_{0}$, the values of $G_{0}$ are observed to be $1.90646 \times 10^{-2} \mathrm{GeV}^{4}, 1.9119 \times$ $10^{-2} \mathrm{GeV}^{4}, 1.91755 \times 10^{-2} \mathrm{GeV}^{4}$, and $1.92 \times 10^{-2} \mathrm{GeV}^{4}$ for temperatures, $T=0,50,100$, and $150 \mathrm{MeV}$, respectively. For the same values of the temperature, in the absence of finite quark masses, the values of $G_{0}$ are observed to be 2.269 $\times 10^{-2} \mathrm{GeV}^{4}, 2.2771 \times 10^{-2} \mathrm{GeV}^{4}, 2.2857 \times 10^{-2} \mathrm{GeV}^{4}$, and $2.29 \times 10^{-2} \mathrm{GeV}^{4}$ for $\rho_{B}=\rho_{0}$. For baryon density, $\rho_{B}=4 \rho_{0}$, the values of $G_{0}$ are given as $1.7367 \times 10^{-2} \mathrm{GeV}^{4}(2.06 \times$ $\left.10^{-2} \mathrm{GeV}^{4}\right), 1.74612 \times 10^{-2} \mathrm{GeV}^{4}\left(2.0713 \times 10^{-2} \mathrm{GeV}^{4}\right), 1.7656$ $\times 10^{-2} \mathrm{GeV}^{4}\left(2.094 \times 10^{-2} \mathrm{GeV}^{4}\right)$, and $1.78 \times 10^{-2} \mathrm{GeV}^{4}(2.112$ $\left.\times 10^{-2} \mathrm{GeV}^{4}\right)$ for values of temperature, $T=0,50,100$, and $150 \mathrm{MeV}$, respectively, for the cases of the finite (zero) quark masses in the trace anomaly.

It may be noted that in the above discussion the finite temperature effects of the nuclear medium on the values of quark and gluon condensates are evaluated through the temperature dependence of scalar fields $\sigma$ and $\zeta$ and the dilaton field $\chi$. 
In literature the scalar quark and gluon condensates at finite temperature are evaluated due to contribution from pion bath using (25) and (26), respectively [42]. Using $m_{q}\langle\bar{q} q\rangle_{0}=$ $-(0.11 \mathrm{GeV})^{4}$ and $\left\langle\left(\alpha_{s} / \pi\right) G^{2}\right\rangle=0.005 \mathrm{GeV}^{4}$ [42] we calculate the quark and gluon condensates at finite temperature and zero baryon density using (25) and (26), respectively. The values of scalar quark condensates are observed to be $-1.824 \times$ $10^{-2},-1.751 \times 10^{-2}$, and $-1.566 \times 10^{-2} \mathrm{GeV}^{3}$ at temperatures $T=50,100$, and $150 \mathrm{MeV}$, respectively. These values of scalar quark condensates can be compared to the values $-1.4014 \times$ $10^{-2},-1.4006 \times 10^{-2}$, and $-1.3628 \times 10^{-2} \mathrm{GeV}^{3}$ at $T=$ 50,100 , and $150 \mathrm{MeV}$ calculated in our present approach where finite temperature effects were considered through scalar fields $\sigma, \zeta$, and $\chi$. Similarly, the values of scalar gluon condensates calculated using (26) are found to be $0.5 \times 10^{-2}$, $0.4994 \times 10^{-2}$, and $0.497 \times 10^{-2} \mathrm{GeV}^{4}$ at $T=50,100$, and $150 \mathrm{MeV}$, respectively. However, for zero baryon density, using the chiral SU(3) model the values of scalar gluon condensates for finite (zero) quark mass term are observed to be $1.94264 \times 10^{-2}\left(2.34550 \times 10^{-2}\right), 1.94269 \times 10^{-2}$ $\left(2.3454 \times 10^{-2}\right)$, and $1.94456 \times 10^{-2}\left(2.3437 \times 10^{-2}\right) \mathrm{GeV}^{4}$ at temperatures $T=50,100$, and $150 \mathrm{MeV}$, respectively. From above discussion we observe that as a function of temperature there are very small variations in the values of scalar quark and gluon condensates in the nuclear medium. This observation agrees, for example, with [47], where gluon and chiral condensates were studied at finite temperature with an effective Lagrangian of pseudoscalar mesons coupled to a scalar glueball. The gluon condensates were found to be very stable up to temperatures of $200 \mathrm{MeV}$, where the chiral sector of the theory reaches its limit of validity [47]. Actually scalar quark and gluon condensates are found to vary effectively with temperature above critical temperature but in hadronic medium, for zero baryon density, these are not much sensitive to temperature effects $[48,49]$.

Note that in the above discussion of scalar gluon and quark condensates, calculated using (1) and (22), respectively, we considered the vacuum values of decay constants $f_{\pi}$ and $f_{K}$ as well as masses $m_{\pi}$ and $m_{K}$ of pions and kaons, respectively. Now we will discuss the effect of medium modified values of $f_{\pi}, f_{K}, m_{\pi}$, and $m_{K}$ on the scalar condensates. In the chiral effective model the pion and kaon decay constants are related to the scalar fields $\sigma$ and $\zeta$ through relations $[5,12]$

$$
\begin{gathered}
f_{\pi}=-\sigma, \\
f_{K}=-\frac{1}{2}(\sigma+\sqrt{2} \zeta),
\end{gathered}
$$

respectively. From the above relations it is clear that the medium dependence of scalar fields $\sigma$ and $\zeta$ can be used to study the density and temperature dependence of decay constants of pions and kaons. For example, using (27), at zero temperature the values of pion decay constant are observed to be $93.3,59.75$, and $42.99 \mathrm{MeV}$ at baryonic densities $\rho_{B}=0$, $\rho_{0}$, and $2 \rho_{0}$, respectively. The values of kaons decay constants, using (28), are observed to be $122,98.6$, and $87.93 \mathrm{MeV}$ at $\rho_{B}=0, \rho_{0}$, and $2 \rho_{0}$, respectively. In [50] the masses of pions were calculated in linear density approximation using the chiral perturbation theory. At baryon density $\rho_{B}=\rho_{0}$ and zero temperature the mass of pion changes from the vacuum value $139 \mathrm{MeV}$ to $145.8 \mathrm{MeV}$. The properties of kaons and antikaons had been investigated in the literature using the chiral model [9] and coupled channel approach [51-54]. In the present work, to study the effect of medium modified masses of kaons on the values of quark and gluon condensates we will use the chiral SU(3) model [9]. In the nuclear medium the kaons feel repulsive interactions and their in-medium mass increases as a function of density whereas the antikaons feel attractive interactions and their in-medium masses drop as we move to higher baryon density. For the present purpose considering the average mass of kaons and antikaons, the in-medium mass at temperature $T=0$ is observed to be $494,488.88$, and $466.08 \mathrm{MeV}$ at $\rho_{B}=0, \rho_{0}$, and $2 \rho_{0}$, respectively. Taking into account the above discussed inmedium properties of $f_{\pi}, f_{K}, m_{\pi}$, and $m_{K}$, at baryonic density $\rho_{B}=\rho_{0}$ and zero temperature, the values of scalar quark and gluon condensates are observed to be $-0.6222 \times 10^{-2} \mathrm{GeV}^{3}$ and $1.9869 \times 10^{-2} \mathrm{GeV}^{4}$, respectively. These values of scalar quark and gluon condensates can be compared to $-0.8829 \times$ $10^{-2} \mathrm{GeV}^{3}$ and $1.9065 \times 10^{-2} \mathrm{GeV}^{4}$, respectively which were calculated without the medium modification of $f_{\pi}, f_{K}, m_{\pi}$, and $m_{K}$. We conclude that the medium modification of $f_{\pi}$, $f_{K}, m_{\pi}$, and $m_{K}$ causes more decrease in the values of scalar quark and gluon condensates at finite baryonic density.

Now we will calculate the in-medium masses of scalar, vector, and axial-vector mesons using the values of condensates from chiral SU(3) model. In Figure 3, the subfigures (a), (c), and (e) show the variation of the mass shift of scalar mesons $D_{0}$ as a function of square of the Borel mass parameter, $M$, at nuclear matter densities $\rho_{0}, 2 \rho_{0}$, and $4 \rho_{0}$, respectively. The subfigures (b), (d), and (f) show the variation of the scalar mesons $B_{0}$ as a function of square of the Borel mass parameter, $M$, at nuclear matter densities $\rho_{0}$, $2 \rho_{0}$, and $4 \rho_{0}$, respectively. In each subplot we have shown the results at temperatures $T=0,50,100$, and $150 \mathrm{MeV}$. We observe that for scalar mesons, $D_{0}$, at temperature, $T$ $=0$, the values of mass shift are found to be 76,114, and $148 \mathrm{MeV}$ at baryon densities $\rho_{0}, 2 \rho_{0}$, and $4 \rho_{0}$, respectively. For temperature $T=50 \mathrm{MeV}$ the values of mass shift are observed to be 71,109 , and $144 \mathrm{MeV}$ at baryon densities $\rho_{0}, 2 \rho_{0}$, and $4 \rho_{0}$, respectively. At temperature $T=100 \mathrm{MeV}$ the above values of mass shift are observed to be 66,103 , and $139 \mathrm{MeV}$, whereas at $T=150 \mathrm{MeV}$ the values of mass shift changes to 58,94 , and $131 \mathrm{MeV}$ at baryon densities $\rho_{0}, 2 \rho_{0}$, and $4 \rho_{0}$, respectively. From the above discussion we conclude that for a given value of temperature the mass shift of scalar mesons, $D_{0}$, increases as a function of density of the nuclear medium. On the other hand as a function of temperature of the nuclear medium, for a constant value of density, the mass shift of scalar mesons, $D_{0}$, decreases.

As we can see from Figure 3 the values of mass shift of scalar $B_{0}$ mesons also increases as a function of density of the nuclear medium. At temperature, $T=0$, the values of mass shift are observed to 224,334 , and $420 \mathrm{MeV}$ at baryon densities $\rho_{0}, 2 \rho_{0}$, and $4 \rho_{0}$, respectively. At temperature $T=$ $50 \mathrm{MeV}$ the above values of mass shift are found to be 211, 321, 


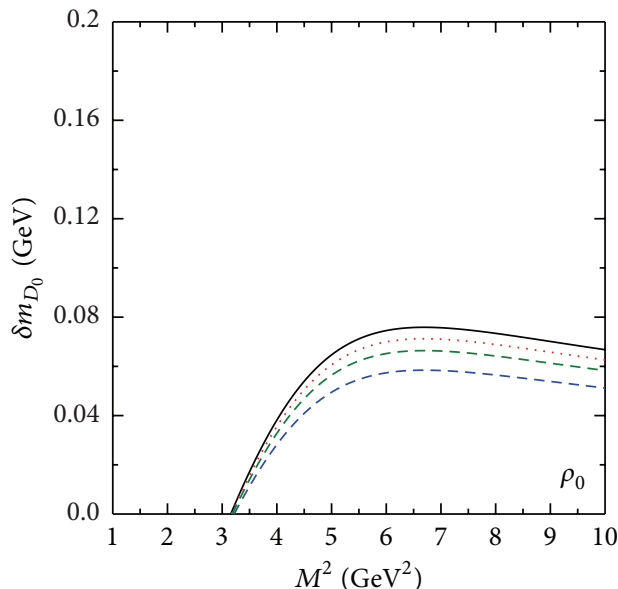

(a)

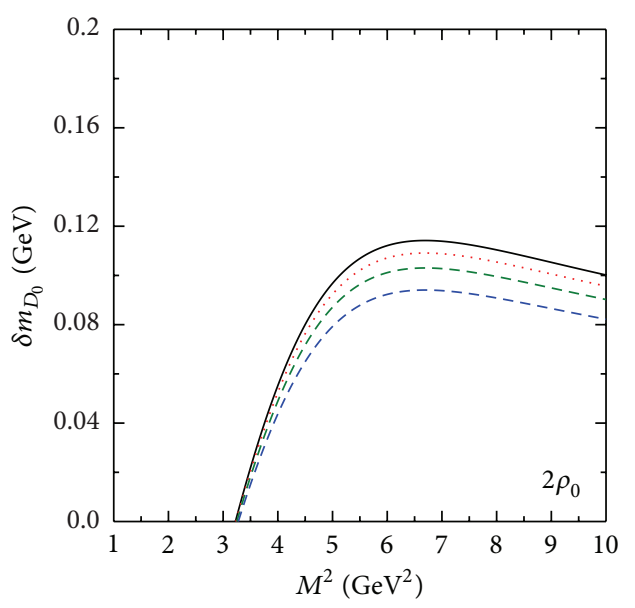

(c)

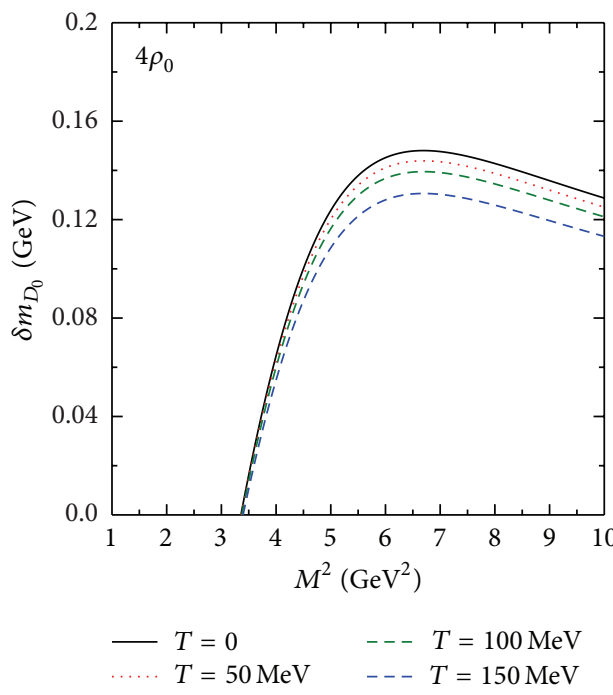

(e)

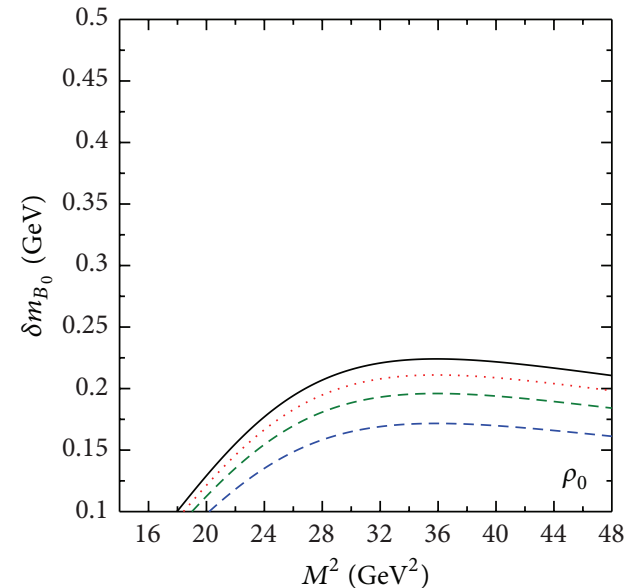

(b)

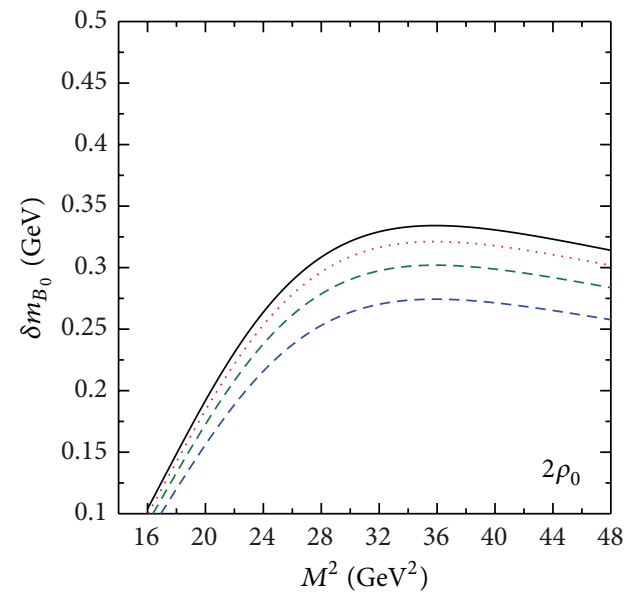

(d)

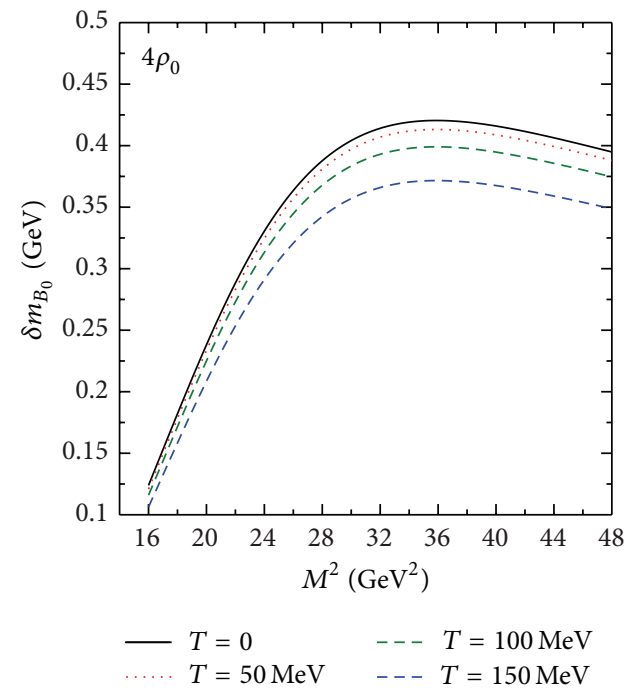

(f)

Figure 3: (Color online) Figure shows the variation of the mass shift of scalar mesons $D_{0}$ (subplots (a), (c), and (e)) and $B_{0}$ (subplots (b), (d), and (f)) as a function of the squared Borel mass parameter, $M^{2}$. We show the results at baryon densities $\rho_{0}, 2 \rho_{0}$, and $4 \rho_{0}$. For each value of baryon density the results are shown at temperatures, $T=0,50,100$, and $150 \mathrm{MeV}$. Also the medium modifications of decay constants and masses of pions and kaons are considered while evaluating the above shown mass-shift of scalar mesons. 
and $413 \mathrm{MeV}$ at baryon densities $\rho_{0}, 2 \rho_{0}$, and $4 \rho_{0}$, respectively. For temperature $T=100 \mathrm{MeV}$ the values of mass shift are found to be 196,302 , and $399 \mathrm{MeV}$ whereas at $T=150 \mathrm{MeV}$ these values of mass shift changes to 171,274 , and $372 \mathrm{MeV}$ at $\rho_{0}, 2 \rho_{0}$, and $4 \rho_{0}$, respectively. For a given value of nuclear matter density the values of mass shift for the $B_{0}$ mesons are found to decrease with the increase in the temperature of the medium.

We also calculate the values of scattering lengths of scalar mesons using (10) for different values of density and temperature of the medium. At temperature $T=0$ and baryon densities, $\rho_{B}=\rho_{0}$ and $4 \rho_{0}$, the values of scattering lengths for scalar mesons $D_{0}\left(B_{0}\right)$ are observed to be $1.42(5.05)$ and $0.70(2.41) \mathrm{fm}$, respectively. For temperature $T=100 \mathrm{MeV}$ and baryon densities, $\rho_{B}=\rho_{0}$ and $4 \rho_{0}$, the values of scattering lengths for $D_{0}\left(B_{0}\right)$ are observed to be 1.23 (4.40) and 0.66 (2.28) fm, respectively. We note that the value of scattering length decreases as a function of density and temperature of the nuclear medium. As discussed above the scattering lengths are evaluated using (10). In this equation we have the parameter $a$ which is directly proportional to the scattering length of mesons. As discussed earlier the value of parameter $a$ is evaluated by solving simultaneously (16) and the equation obtained by differentiate (16) w.r.t. $1 / M^{2}$. The magnitude of parameter $a$ is found to decrease as we move from low to higher value of baryonic density or from zero to finite value of temperature of the medium. This behavior of parameter $a$ as a function of density and temperature of the medium results in the similar changes in the values of scattering lengths of scalar mesons.

Figure 4 shows the variation of the mass shift of vector mesons $D^{*}$ and $B^{*}$ as a function of square of the Borel mass parameter. Here also we have shown the results at nuclear matter densities $\rho_{0}, 2 \rho_{0}$, and $4 \rho_{0}$. We observe that at nuclear matter density $\rho_{B}=\rho_{0}$ the values of mass shift for vector mesons, $D^{*}$ are observed to be $-76,-71,-65$, and $-56 \mathrm{MeV}$ at temperatures $T=0,50,100$, and $150 \mathrm{MeV}$, respectively. For baryon density $\rho_{B}=2 \rho_{0}$ the values of mass shift are found to be $-111,-106,-98$, and $-87 \mathrm{MeV}$, whereas for $\rho_{B}=4 \rho_{0}$ the values of the mass shift changes to $-128,-125,-120$, and $-108 \mathrm{MeV}$ at temperatures $T=0,50,100$, and $150 \mathrm{MeV}$, respectively. Note that for a given value of density, the magnitude of the mass shift of vector mesons decreases as a function of temperature of the nuclear medium. On the other hand as a function of density of the medium the magnitude of the mass shift of the vector mesons $D^{*}$ increases. For nuclear matter saturation density the values of mass shift for the $B^{*}$ mesons are found to be $-366,-344,-311$, and $-275 \mathrm{MeV}$ at temperatures $T=0,50,100$, and $150 \mathrm{MeV}$, respectively. At baryon density $2 \rho_{0}$ the above values of mass shift changes to $-557,-534,-498$, and $-447 \mathrm{MeV}$ at temperatures $T=0$, 50,100 , and $150 \mathrm{MeV}$, respectively, whereas for baryon density $4 \rho_{0}$ the above values of mass shift are found to be -701 , $-689,-662$, and $-609 \mathrm{MeV}$ at temperatures $T=0,50,100$, and $150 \mathrm{MeV}$, respectively. At temperature $T=0$ and baryon densities, $\rho_{B}=\rho_{0}$ and $4 \rho_{0}$, the values of scattering lengths for vector mesons $D^{*}\left(B^{*}\right)$ are observed to be $-1.31(-7.72)$ and $-0.54(-3.58) \mathrm{fm}$, respectively. For temperature $T=100 \mathrm{MeV}$ and baryon densities, $\rho_{B}=\rho_{0}$ and $4 \rho_{0}$, the values of scattering lengths for $D^{*}\left(B^{*}\right)$ are observed to be $-1.12(-6.72)$ and -0.51 $(-3.54) \mathrm{fm}$, respectively. We observe that the magnitude of the scattering lengths of vector mesons decreases in moving from low to higher value of density or temperature of the medium.

In Figure 5, for given values of temperatures and densities we have shown the variation of mass shift of axial-vector mesons $D_{1}$ and $B_{1}$ as a function of square of the Borel mass parameter. We observe that at baryon density, $\rho_{B}=\rho_{0}$, the values of mass shift for axial-vector meson $D_{1}$ are observed to be $73,69,63$, and $55 \mathrm{MeV}$ at temperatures, $T=0,50,100$, and $150 \mathrm{MeV}$. At baryon density $2 \rho_{0}\left(4 \rho_{0}\right)$ the values of mass shift are found to be 108 (131), 104(128), 97(123), and 87(113) at temperatures, $T=0,50,100$, and $150 \mathrm{MeV}$. For the axialvector meson $B_{1}$ at baryon density $\rho_{0}\left(2 \rho_{0}\right)$ the values of mass shift are found to be 267 (396), 251(381), 233(357), and 203(324) $\mathrm{MeV}$ at temperatures, $T=0,50,100$, and $150 \mathrm{MeV}$, respectively. At baryon density $4 \rho_{0}$ the values of mass shift are found to be $492,485,467$, and $434 \mathrm{MeV}$ at temperatures, $T=$ $0,50,100$, and $150 \mathrm{MeV}$, respectively. The values of scattering lengths for axial-vector mesons $D_{1}\left(B_{1}\right)$ at temperature $T=$ 0 and baryon densities, $\rho_{B}=\rho_{0}$ and $4 \rho_{0}$, are observed to be 1.38 (6.02) and $0.62(2.83) \mathrm{fm}$, respectively. For temperature $T=100 \mathrm{MeV}$ and baryon densities, $\rho_{B}=\rho_{0}$ and $4 \rho_{0}$, the values of scattering lengths for $D^{*}\left(B^{*}\right)$ changes to 1.19 (5.25) and $0.58(2.69) \mathrm{fm}$, respectively. From the above discussions we observe a positive value of mass shift for scalar $\left(D_{0}, B_{0}\right)$ and axial-vector mesons $\left(D_{1}, B_{1}\right)$ in the nuclear medium. However, the values of mass shift for vector mesons $\left(D^{*}, B^{*}\right)$ are found to be negative. It means the masses of above scalar and axial-vector mesons in the nuclear medium may be large compared to the value in free space and this may lead to a decrease in the yield of these mesons in heavy-ion collisions.

Now we will discuss the effect of different individual condensates on the in-medium modification of scalar $\left(D_{0}\right.$, $\left.B_{0}\right)$, vector $\left(D^{*}, B^{*}\right)$, and axial-vector $\left(D_{1}, B_{1}\right)$ mesons. In Figures 6,7 , and 8 we compare the contributions of individual condensates to the mass shift of scalar mesons, $D_{0}$, vector mesons, $D^{*}$, and axial-vector mesons, $D_{1}$, respectively. The subplots (a), (c), and (e) show the results at temperature $T=0$, whereas the subplots (b), (d), and (f) are plotted for temperature $T=100 \mathrm{MeV}$. We have shown the results at baryon densities $\rho_{0}, 2 \rho_{0}$, and $4 \rho_{0}$. Note that in Figure 6 and in the subsequent figures of this paper the word "Total" is for the contribution of all condensates to the properties of mesons. Similarly, "Quark,", "Quark,", "Quark," and "Gluon," are denoting the contribution of $\langle\bar{q} q\rangle,\left\langle q^{\dagger} i D_{0} q\right\rangle,\left\langle\bar{q} i D_{0} i D_{0} q\right\rangle$, and $\left\langle\alpha_{s} G G / \pi\right\rangle$, respectively, to the in-medium properties of mesons.

We observe that at temperature $T=0$; if we consider the contribution of scalar quark condensates, only then the values of mass shift for the scalar $D_{0}$ mesons are found to be 73.30 and $150.68 \mathrm{MeV}$ at nuclear matter density $\rho_{B}=$ $\rho_{0}$ and $4 \rho_{0}$, respectively. When we consider the individual contributions of $\left\langle q^{\dagger} i D_{0} q\right\rangle,\left\langle\bar{q} i D_{0} i D_{0} q\right\rangle$, and $\left\langle\alpha_{s} G G / \pi\right\rangle$ condensates then the values of mass-shift at $\rho_{0}\left(4 \rho_{0}\right)$ and $T=0$ are observed to be 4.01(16.07), 12.70(37.87), and 6.68(25.96) MeV, 


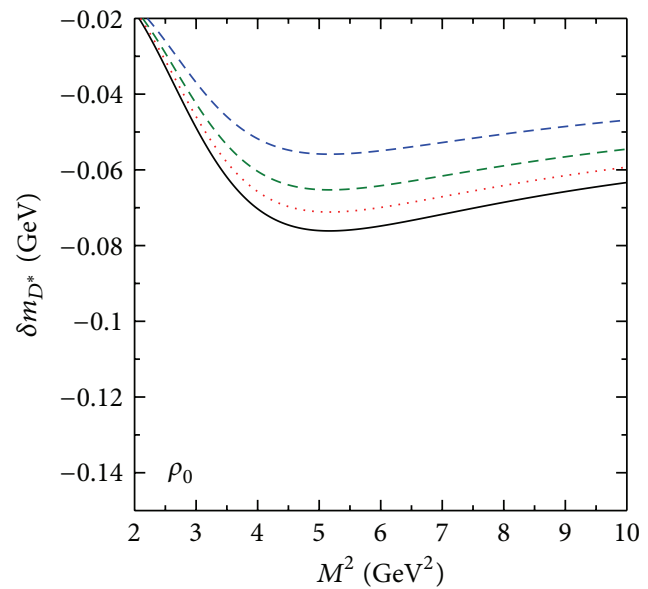

(a)

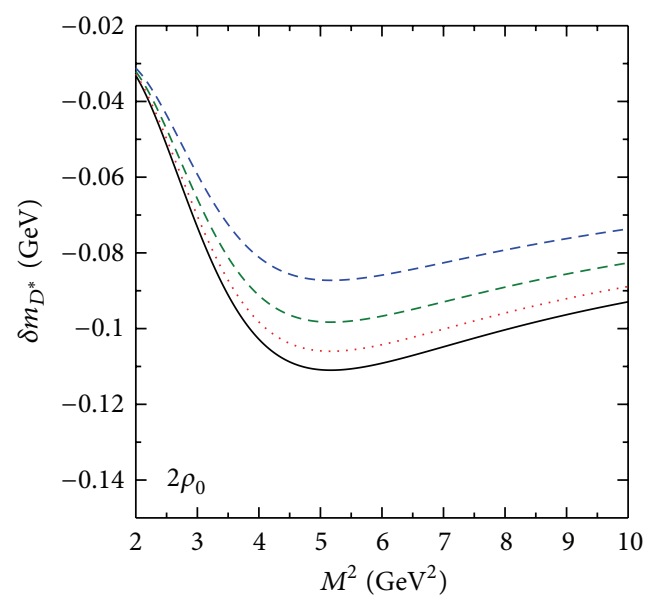

(c)

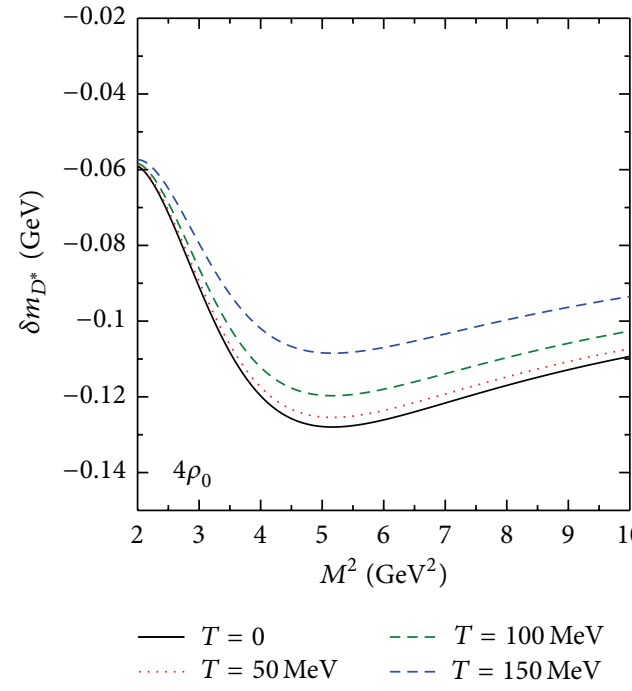

(e)

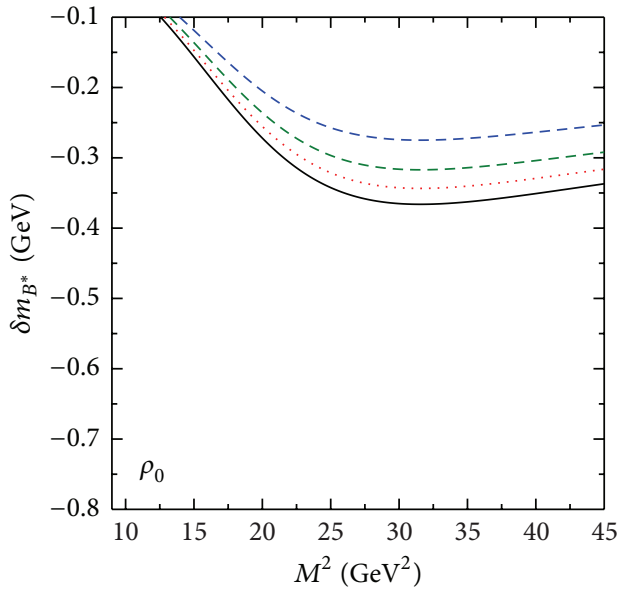

(b)

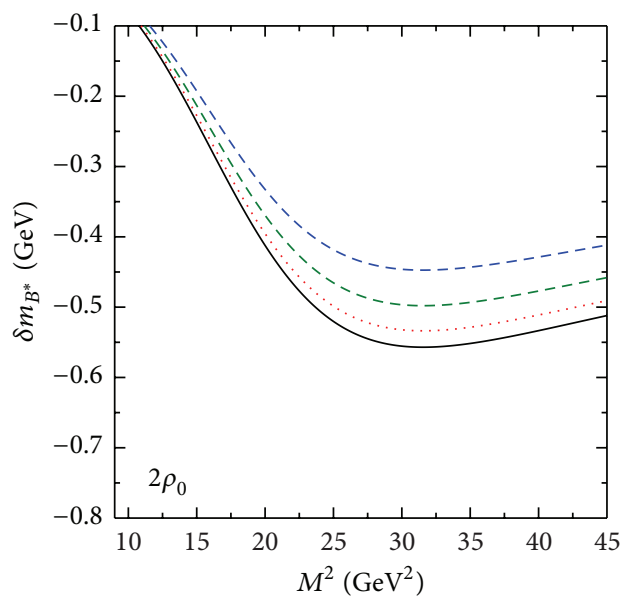

(d)

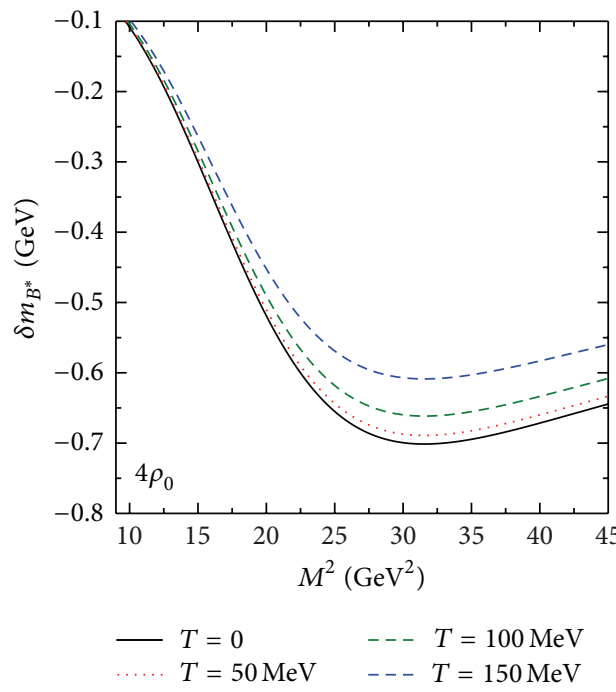

(f)

Figure 4: (Color online) Figure shows the variation of the mass shift of vector mesons $D^{*}$ (subplots (a), (c), and (e)) and $B^{*}$ (subplots (b), (d), and (f)) as a function of the squared Borel mass parameter, $M^{2}$. We have shown the results at baryon densities $\rho_{0}, 2 \rho_{0}$, and $4 \rho_{0}$. For each value of the baryon density the results are shown at temperatures, $T=0,50,100$, and $150 \mathrm{MeV}$. 


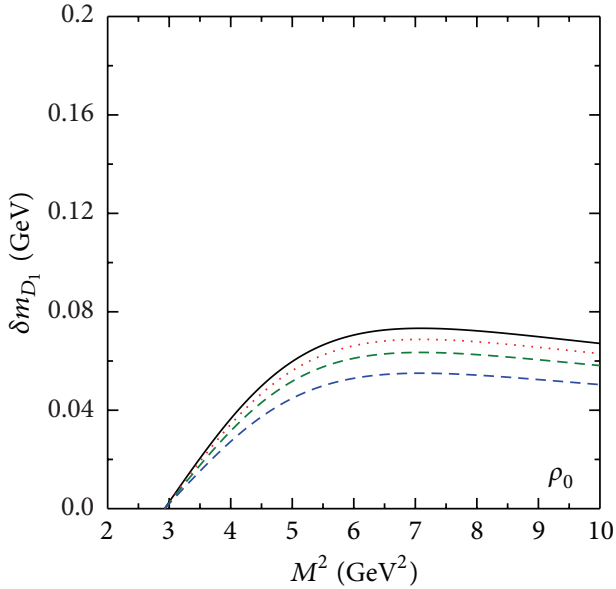

(a)

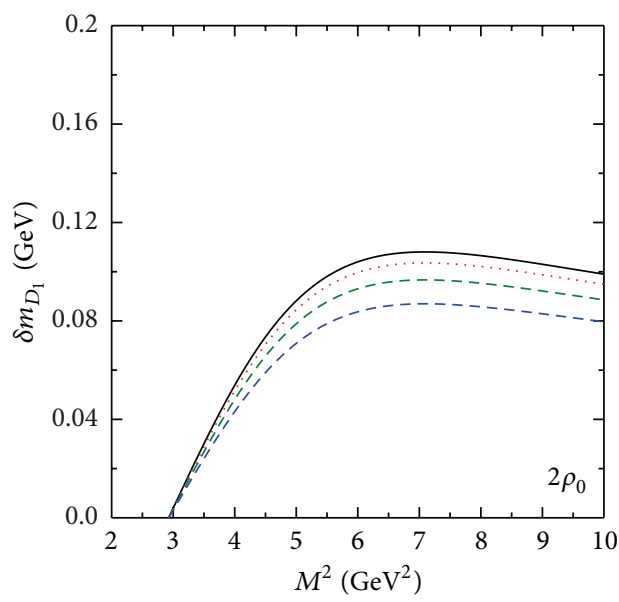

(c)

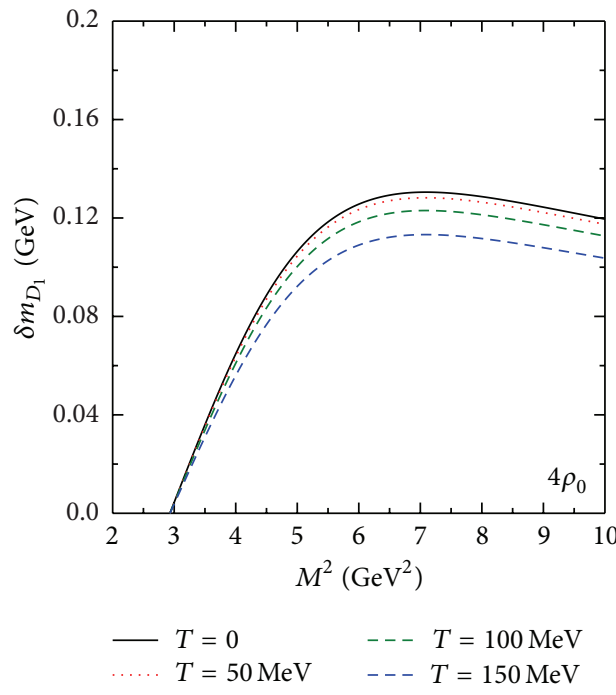

(e)

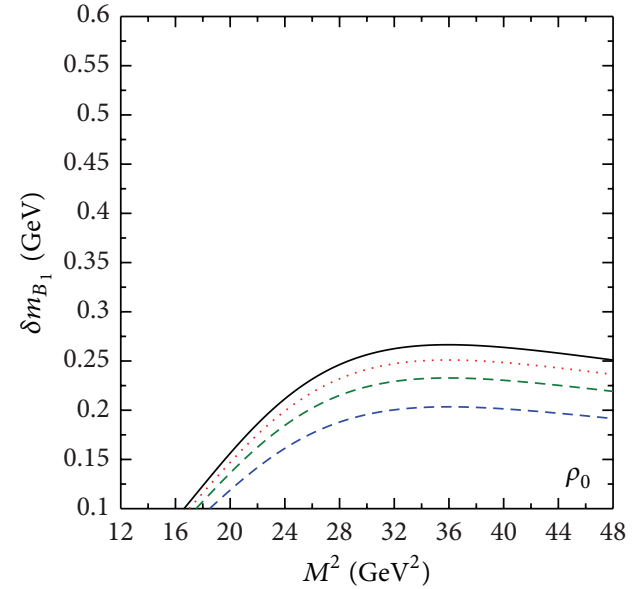

(b)

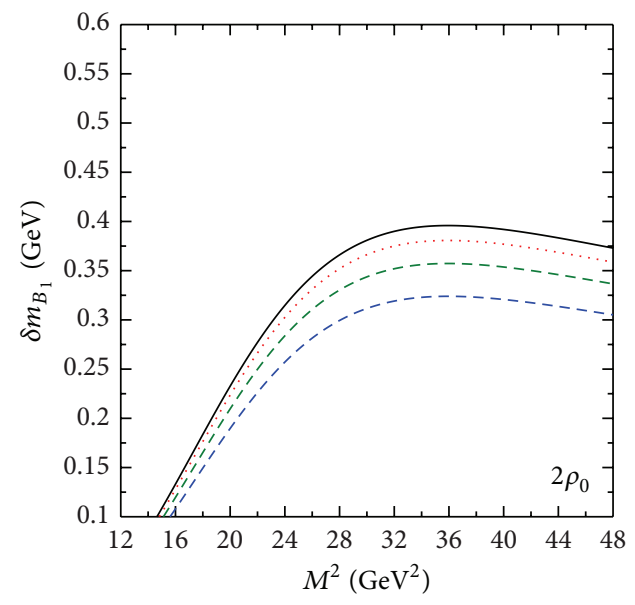

(d)

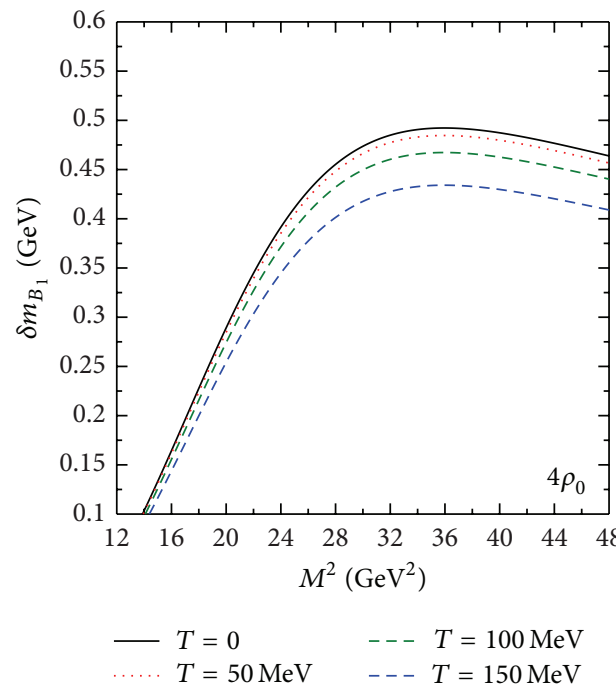

(f)

FIGURE 5: (Color online) Figure shows the variation of the mass shift of axial-vector mesons $D_{1}$ (subplots (a), (c), and (e)) and $B_{1}$ (subplots (b), (d), and (f)) as a function of the squared Borel mass parameter, $M^{2}$. We have shown the results at baryon densities $\rho_{0}, 2 \rho_{0}$, and $4 \rho_{0}$. For each value of the baryon density the results are shown at temperatures, $T=0,50,100$, and $150 \mathrm{MeV}$. 


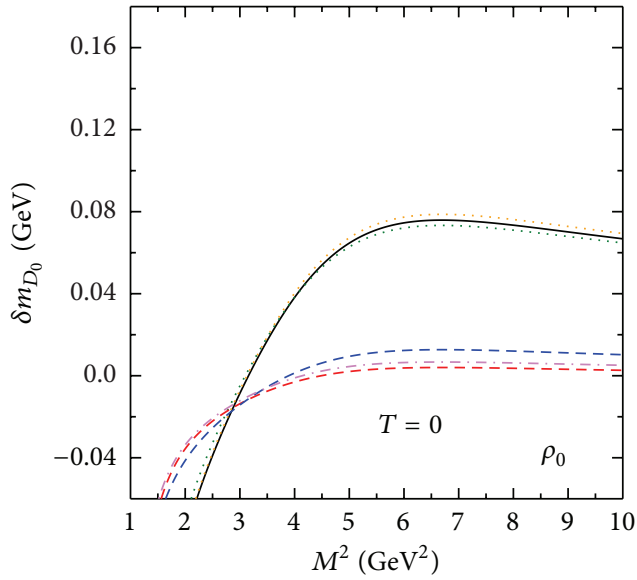

(a)

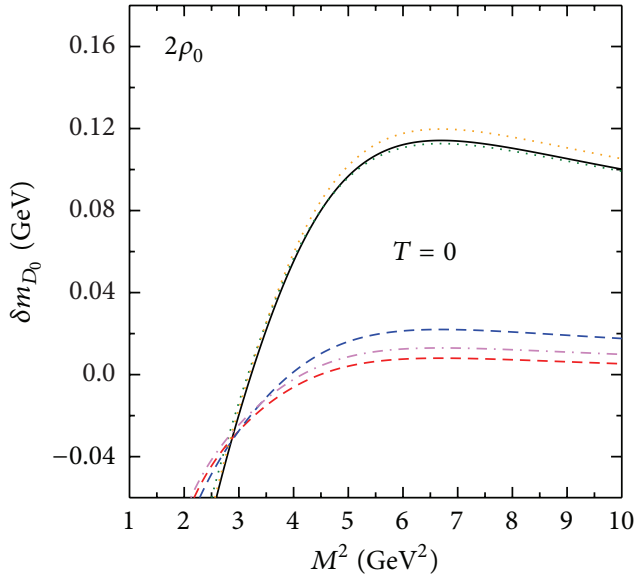

(c)

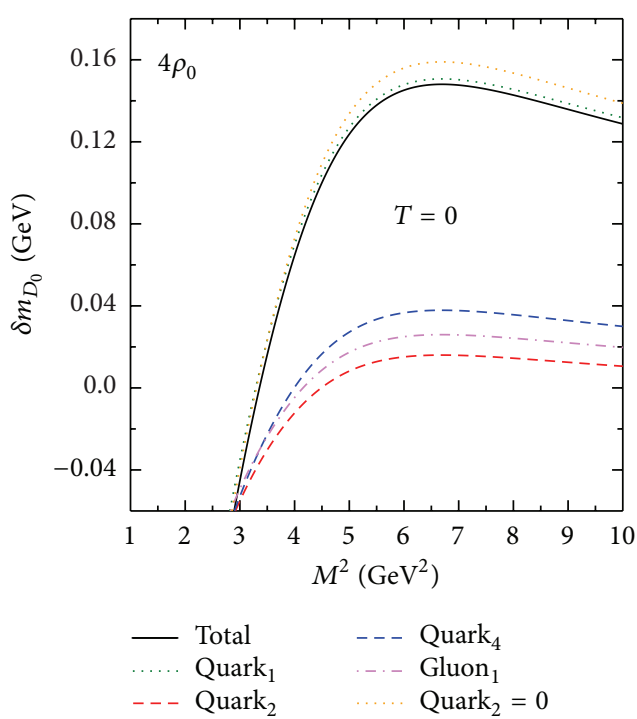

(e)

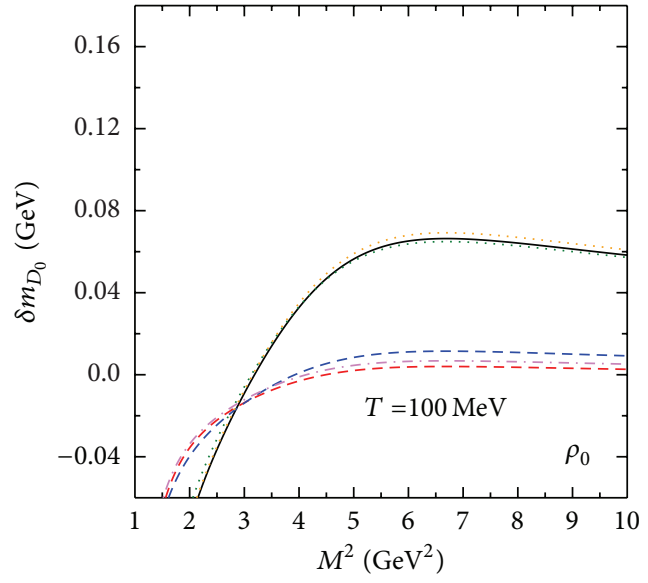

(b)

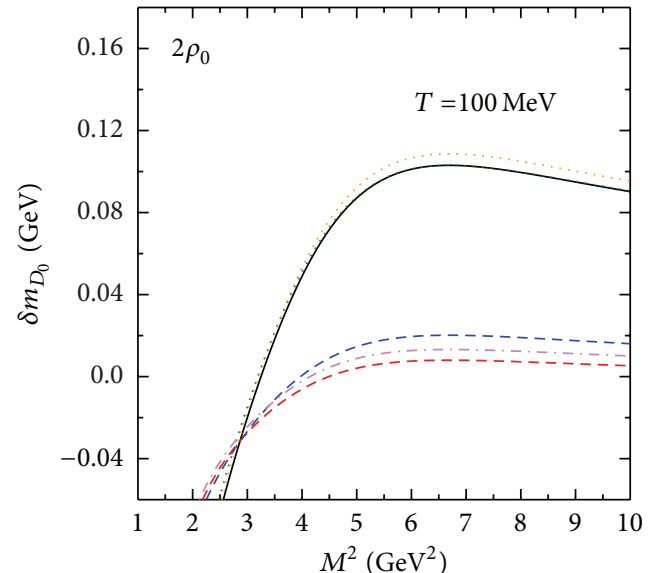

(d)

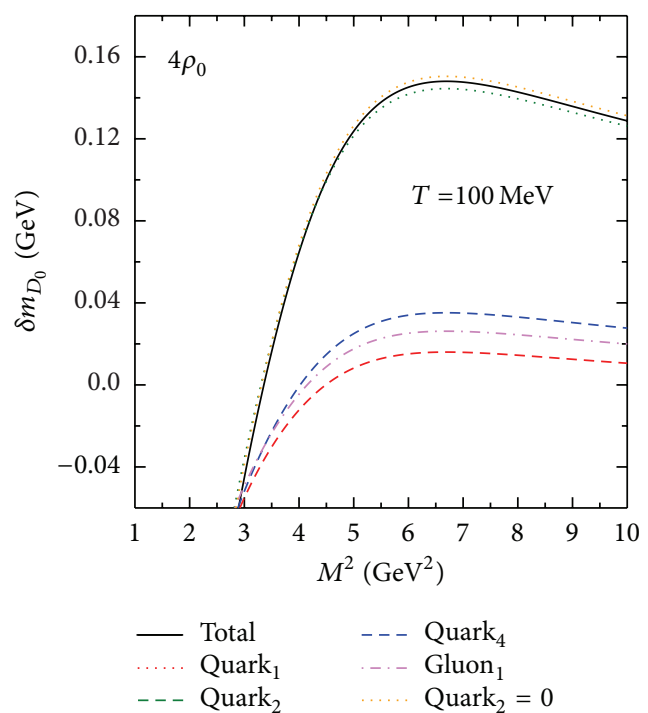

(f)

Figure 6: (Color online) Figure shows the contribution of individual condensates to the mass shift of scalar mesons $D_{0}$ as a function of the squared Borel mass parameter, $M^{2}$. The subplots (a), (c), and (e) show the results at temperature $T=0$, whereas the subplots (b), (d), and (f) are plotted for temperature $T=100 \mathrm{MeV}$. We have shown the results at baryon densities $\rho_{0}, 2 \rho_{0}$, and $4 \rho_{0}$. 


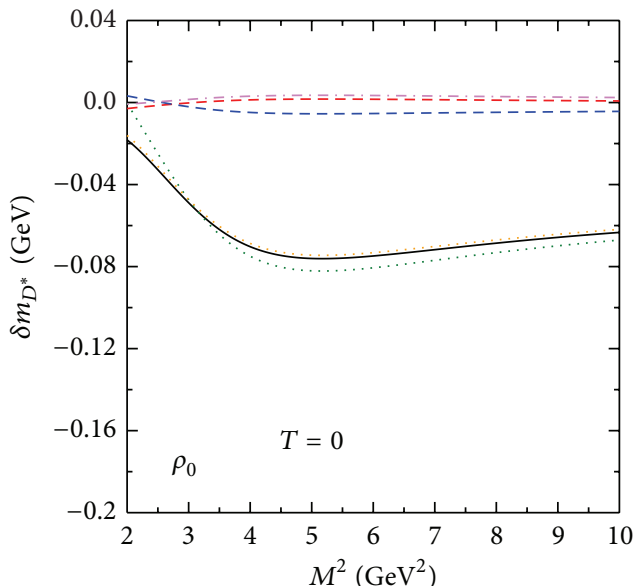

(a)

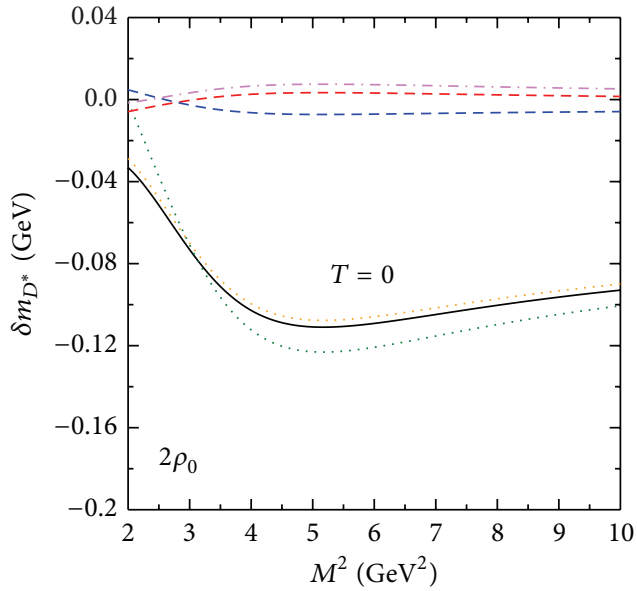

(c)

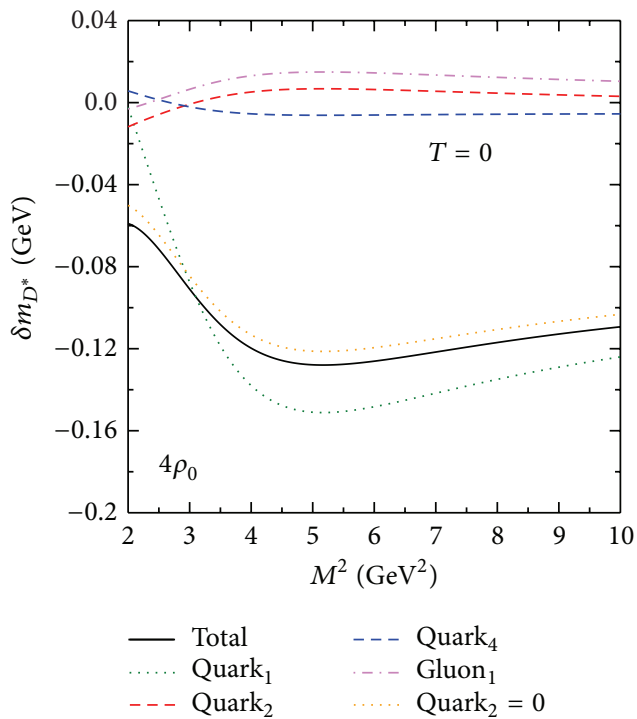

(e)

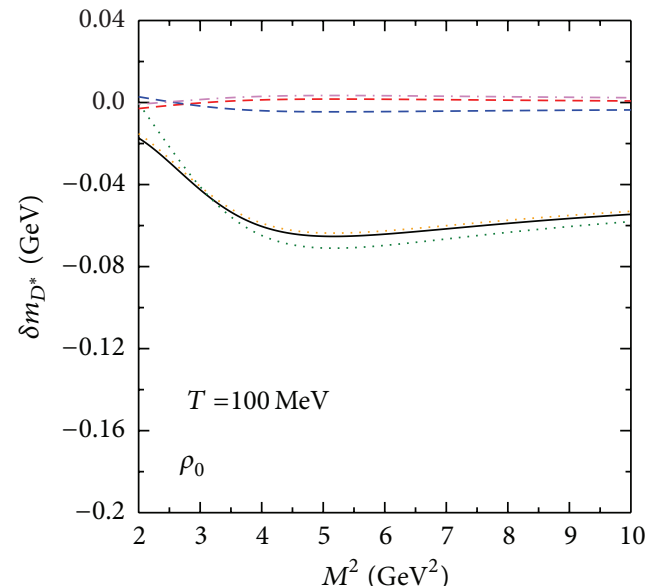

(b)

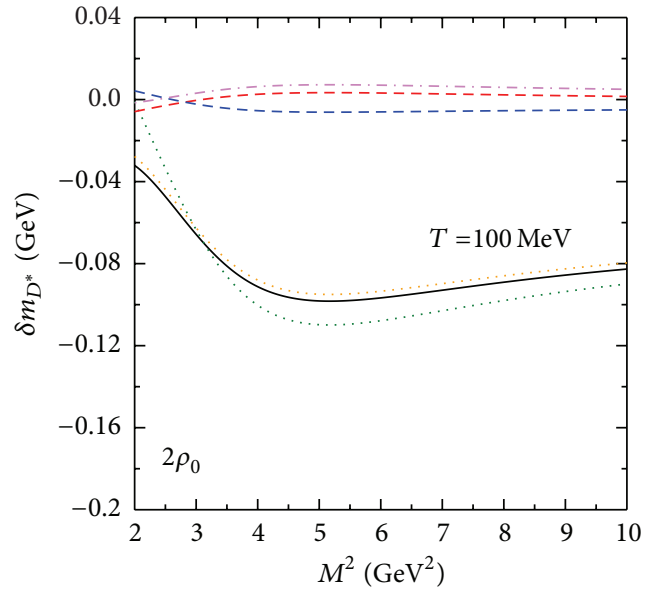

(d)

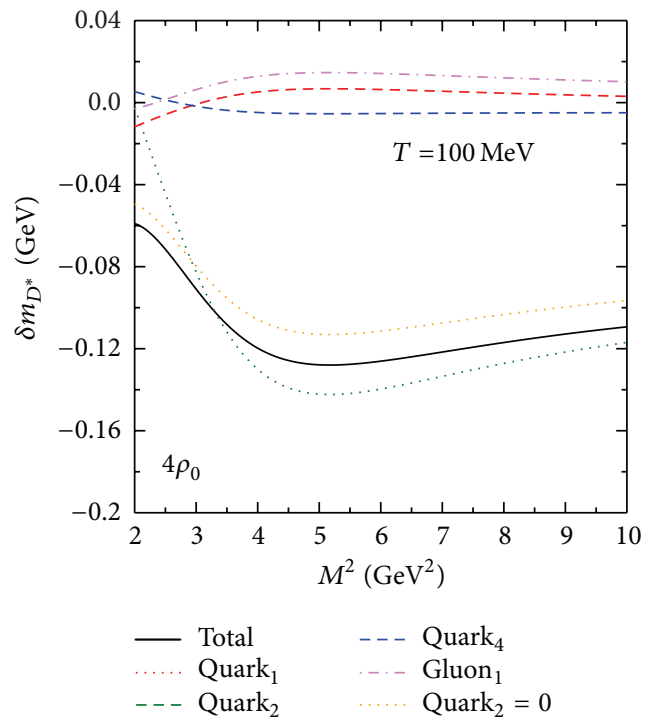

(f)

FIgURE 7: (Color online) Figure shows the contribution of individual condensates to the mass shift of vector mesons $D^{*}$ as a function of the squared Borel mass parameter, $M^{2}$. The subplots (a), (c), and (e) show the results at temperature $T=0$, whereas the subplots (b), (d), and (f) are plotted for temperature $T=100 \mathrm{MeV}$. We have shown the results at baryon densities $\rho_{0}, 2 \rho_{0}$, and $4 \rho_{0}$. 


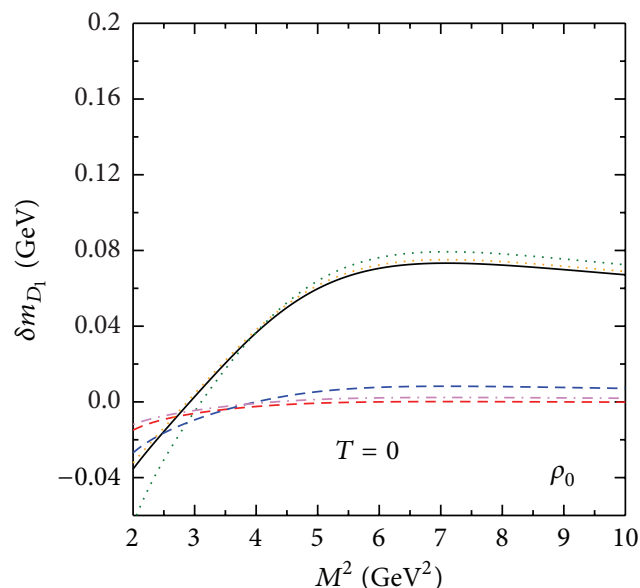

(a)

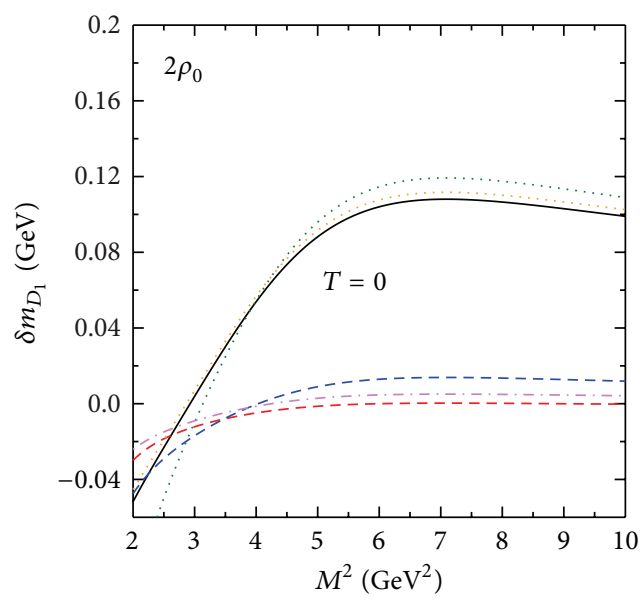

(c)

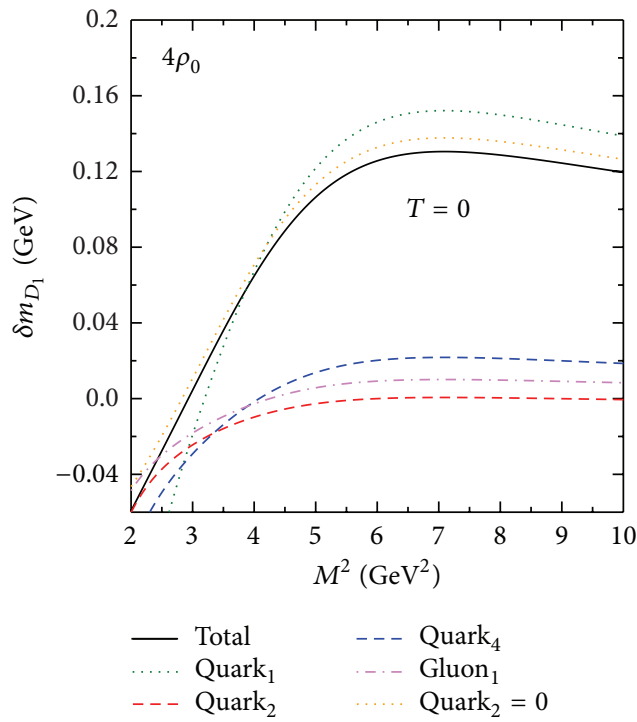

(e)

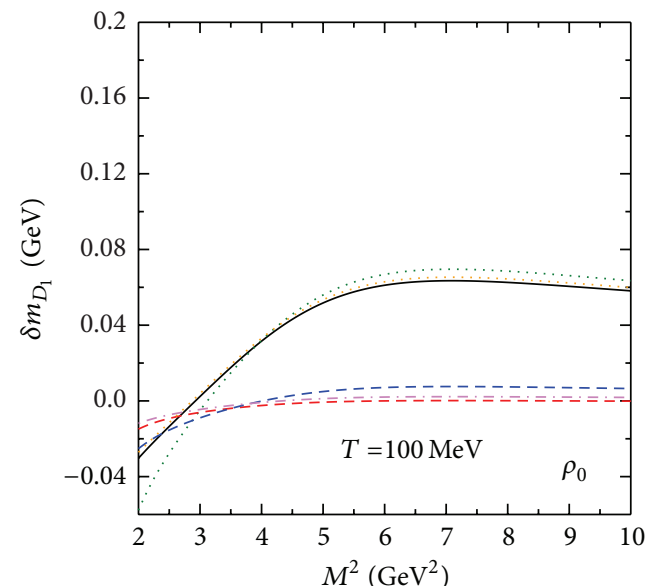

(b)

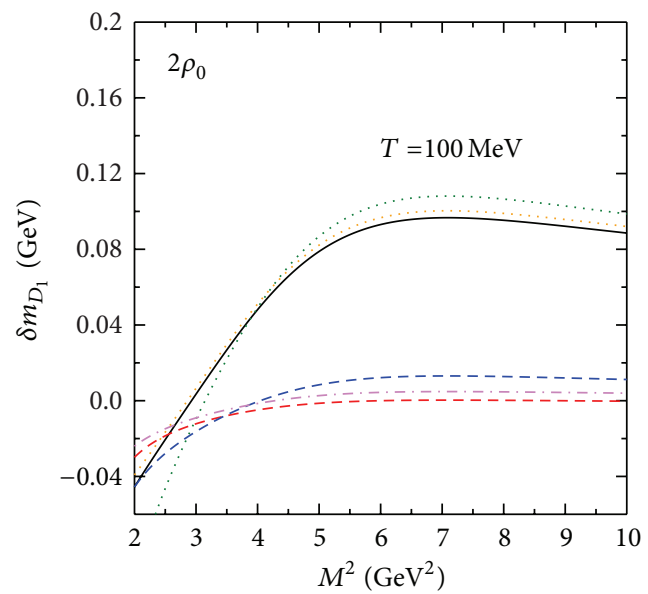

(d)

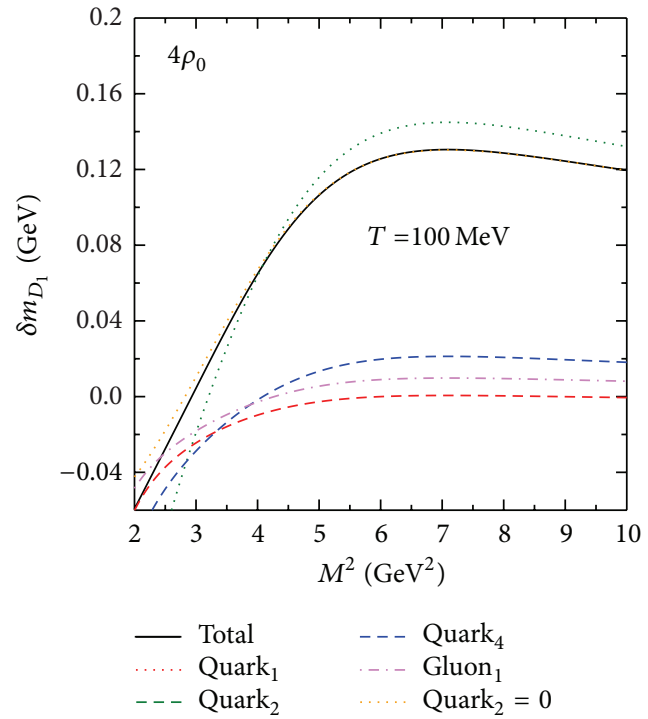

(f)

FIgURE 8: (Color online) Figure shows the contribution of individual condensates to the mass shift of axial-vector mesons $D_{1}$ as a function of the squared Borel mass parameter, $M^{2}$. The subplots (a), (c), and (e) show the results at temperature $T=0$, whereas the subplots (b), (d), and (f) are plotted for temperature $T=100 \mathrm{MeV}$. We have shown the results at baryon densities $\rho_{0}, 2 \rho_{0}$, and $4 \rho_{0}$. 
respectively. From above discussion we observe that the maximum contribution to the in-medium modification of scalar $D_{0}$ mesons is from light quark condensate $\langle\bar{q} q\rangle$. Note that leaving the quark condensate, $\left\langle q^{\dagger} i D_{0} q\right\rangle$, all the other condensates have been evaluated within chiral SU(3) model in the present investigation. So in Figure 6 we also show the variation of the mass shift of scalar $D_{0}$ mesons as a function of squared Borel mass parameter when we neglect the contribution of $\left\langle q^{\dagger} i D_{0} q\right\rangle$ condensate. We observe that if we neglect the contribution of $\left\langle q^{\dagger} i D_{0} q\right\rangle$ condensate then the values of mass shift are found to be 78.70 and $158.99 \mathrm{MeV}$ at densities $\rho_{0}$ and $4 \rho_{0}$, respectively. Note that considering the contribution of all condensates, at temperature $T=$ 0 , the values of mass shift were 75.88 and $148.05 \mathrm{MeV}$ at densities $\rho_{0}$ and $4 \rho_{0}$, respectively. So we observe that if we neglect the condensate $\left\langle q^{\dagger} i D_{0} q\right\rangle$ then there is a percentage change of $4 \%$ and $7 \%$ in the mass shift of $D_{0}$ mesons at densities $\rho_{0}$ and $4 \rho_{0}$, respectively. From Figure 7 , we observe that for vector mesons, $D^{*}$, at temperature $T=0$ and baryon density $\rho_{B}=\rho_{0}\left(4 \rho_{0}\right)$, the values of mass shift due to condensates $\langle\bar{q} q\rangle,\left\langle q^{\dagger} i D_{0} q\right\rangle,\left\langle\bar{q} g_{s} \sigma G q\right\rangle,\left\langle\bar{q} i D_{0} i D_{0} q\right\rangle$, and $\left\langle\alpha_{s} G G / \pi\right\rangle$ are observed to be $-82.15(-151.15), 1.689(6.749)$, 18.95(57.16), -5.49(-6.16), and $3.54(14.93) \mathrm{MeV}$, respectively. For temperature $T=100 \mathrm{MeV}$ the above values of mass shift at baryon density $\rho_{B}=\rho_{0}\left(4 \rho_{0}\right)$ changes to $-73.01(-142.35)$, 1.689 (6.75), 17.85 (56.34), $-4.53(-5.43)$, and $3.43(14.64) \mathrm{MeV}$, respectively. For the axial-vector mesons, $D_{1}$, the values of mass shift due to individual condensates $\langle\bar{q} q\rangle,\left\langle q^{\dagger} i D_{0} q\right\rangle$, $\left\langle\bar{q} g_{s} \sigma G q\right\rangle,\left\langle\bar{q} i D_{0} i D_{0} q\right\rangle$, and $\left\langle\alpha_{s} G G / \pi\right\rangle$ are observed to be 79.31(152), 0.1516(0.607), -8.77(-22.63), 8.27(21.76), and 2.34 (10.08) $\mathrm{MeV}$, respectively. For temperature $T=100 \mathrm{MeV}$ the above values of mass shift at baryon density $\rho_{B}=\rho_{0}\left(4 \rho_{0}\right)$ changes to 70 (145), $0.151(0.607),-7.99(-22.04), 7.58(21.26)$, and 2.23 (9.81), respectively. In Figures 9, 10, and 11 we have shown the contributions of individual condensates to the mass shift of scalar mesons, $B_{0}$, vector mesons, $B^{*}$, and axialvector mesons, $B_{1}$, respectively. The subplots (a), (c), and (e) show the results at temperature $T=0$, whereas the subplots (b), (d), and (f) are plotted for temperature $T=100 \mathrm{MeV}$. We have shown the results at baryon densities $\rho_{0}, 2 \rho_{0}$, and $4 \rho_{0}$. In Tables 1, 2, and 3 we have tabulated the values of mass shift for scalar mesons, $B_{0}$, vector mesons, $B^{*}$ and axial-vector mesons, $B_{1}$, respectively. The values of mass shift have been given at baryon densities $\rho_{0}$ and $4 \rho_{0}$ and temperatures $T=0$ and $100 \mathrm{MeV}$.

In [23], the properties of scalar mesons $D_{0}$ and $B_{0}$ had been studied using QCD sum rules and the observed values of mass shift at nuclear saturation density were 69 and $217 \mathrm{MeV}$, respectively. The properties of $D_{0}$ mesons had also been studied in [25] using coupled channel approach. An extra widening from the already large width of the resonance in free space was observed for the $D_{0}$ meson. The properties of vector mesons $\left(D^{*}\right.$ and $\left.B^{*}\right)$ and axial-vector $\left(D_{1}\right.$ and $\left.B_{1}\right)$ had been studied using QCD sum rules in [24]. For vector mesons $D^{*}$ and $B^{*}$ the values of mass shift were -71 and $-380 \mathrm{MeV}$, respectively, whereas for axial-vector mesons $D_{1}$ and $B_{1}$ these values change to 72 and $264 \mathrm{MeV}$, respectively. The observed positive values of mass shift for $D_{0}$ and $D_{1}$ mesons
TABLE 1: The following table gives the values of mass shift of scalar mesons $B_{0}$ (in $\mathrm{MeV}$ units) due to individual condensates at temperatures $T=0$ and $100 \mathrm{MeV}$. For each value of temperature the values are tabulated for baryon densities $\rho_{B}=\rho_{0}$ and $4 \rho_{0}$.

\begin{tabular}{lcccc}
\hline & \multicolumn{2}{c}{$T=0$} & \multicolumn{2}{c}{$T=100 \mathrm{MeV}$} \\
& $\rho_{B}=\rho_{0}$ & $\rho_{B}=4 \rho_{0}$ & $\rho_{B}=\rho_{0}$ & $\rho_{B}=4 \rho_{0}$ \\
\hline Total & 224 & 420 & 196 & 399 \\
$\langle\bar{q} q\rangle$ & 223 & 422 & 195 & 402 \\
$\left\langle q^{\dagger} i D_{0} q\right\rangle$ & 2.26 & 9.04 & 2.26 & 9.04 \\
$\left\langle\bar{q} i D_{0} i D_{0} q\right\rangle$ & 6.60 & 20.04 & 5.99 & 18.69 \\
$\left\langle\frac{\alpha_{s} G G}{\pi}\right\rangle$ & 3.49 & 13.35 & 3.58 & 13.57 \\
$\left\langle q^{\dagger} i D_{0} q\right\rangle=0$ & 226 & 426 & 197 & 405 \\
\hline
\end{tabular}

TABLE 2: The following table gives the values of mass shift of vector mesons $B^{*}$ (in $\mathrm{MeV}$ units) due to individual condensates at temperatures $T=0$ and $100 \mathrm{MeV}$. For each value of temperature the values are tabulated for baryon densities $\rho_{B}=\rho_{0}$ and $4 \rho_{0}$.

\begin{tabular}{lcccc}
\hline & \multicolumn{2}{c}{$T=0$} & \multicolumn{2}{c}{$T=100 \mathrm{MeV}$} \\
& $\rho_{B}=\rho_{0}$ & $\rho_{B}=4 \rho_{0}$ & $\rho_{B}=\rho_{0}$ & $\rho_{B}=4 \rho_{0}$ \\
\hline Total & -367 & -705.69 & -318.08 & -665.88 \\
$\langle\bar{q} q\rangle$ & -373.11 & -728.58 & -323.61 & -687.98 \\
$\left\langle q^{\dagger} i D_{0} q\right\rangle$ & 1.477 & 5.9057 & 1.477 & 5.9057 \\
$\left\langle\bar{q} g_{s} \sigma G q\right\rangle$ & 12.43 & 36.77 & 11.67 & 36.18 \\
$\left\langle\bar{q} i D_{0} i D_{0} q\right\rangle$ & -3.98 & -6.42 & -3.40 & -5.98 \\
$\left\langle\frac{\alpha_{s} G G}{\pi}\right\rangle$ & 1.64 & 7.56 & 1.51 & 7.21 \\
$\left\langle q^{\dagger} i D_{0} q\right\rangle=0$ & -366.34 & -702.26 & -317.28 & -662.48 \\
\hline
\end{tabular}

TABLE 3: (Color online) The following table gives the values of mass shift of axial vector mesons $B_{1}$ (in $\mathrm{MeV}$ units) due to individual condensates at temperatures $T=0$ and $100 \mathrm{MeV}$. For each value of temperature the values are tabulated for baryon densities $\rho_{B}=\rho_{0}$ and $4 \rho_{0}$.

\begin{tabular}{lcccc}
\hline & \multicolumn{2}{c}{$T=0$} & \multicolumn{2}{c}{$T=100 \mathrm{MeV}$} \\
& $\rho_{B}=\rho_{0}$ & $\rho_{B}=4 \rho_{0}$ & $\rho_{B}=\rho_{0}$ & $\rho_{B}=4 \rho_{0}$ \\
\hline Total & 266.51 & 492.22 & 232.75 & 467.29 \\
$\langle\bar{q} q\rangle$ & 270.11 & 503.57 & 236.35 & 478.86 \\
$\left\langle q^{\dagger} i D_{0} q\right\rangle$ & 0.88 & 3.53 & 0.88 & 3.53 \\
$\left\langle\bar{q} g_{s} \sigma G q\right\rangle$ & -6.34 & -16.79 & -5.83 & -16.40 \\
$\left\langle\bar{q} i D_{0} i D_{0} q\right\rangle$ & 4.79 & 12.53 & 4.39 & 12.23 \\
$\left\langle\frac{\alpha_{s} G G}{\pi}\right\rangle$ & 1.41 & 6.39 & 1.31 & 6.12 \\
$\left\langle q^{\dagger} i D_{0} q\right\rangle=0$ & 266.75 & 493.11 & 232.99 & 468.20 \\
\hline
\end{tabular}

in our present investigation and also in earlier investigations indicate that these mesons feel repulsive interactions in the nuclear medium and their in-medium mass increases as function of baryonic density. This means the chances of decay of higher charmonium states to these heavy charmed mesons pairs are suppressed and hence these mesons may not cause a decrease in the production of $J / \psi$ mesons in heavy-ion 


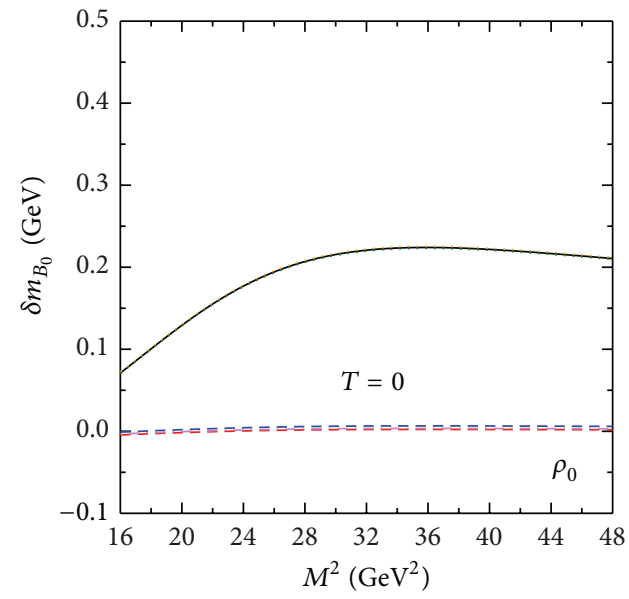

(a)

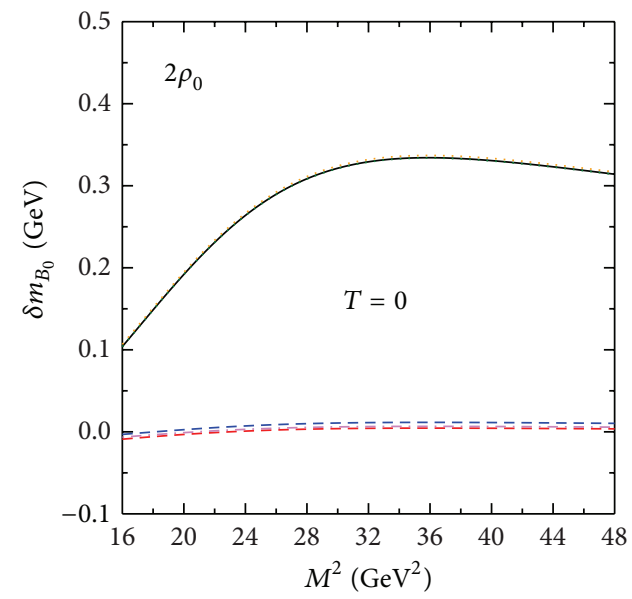

(c)

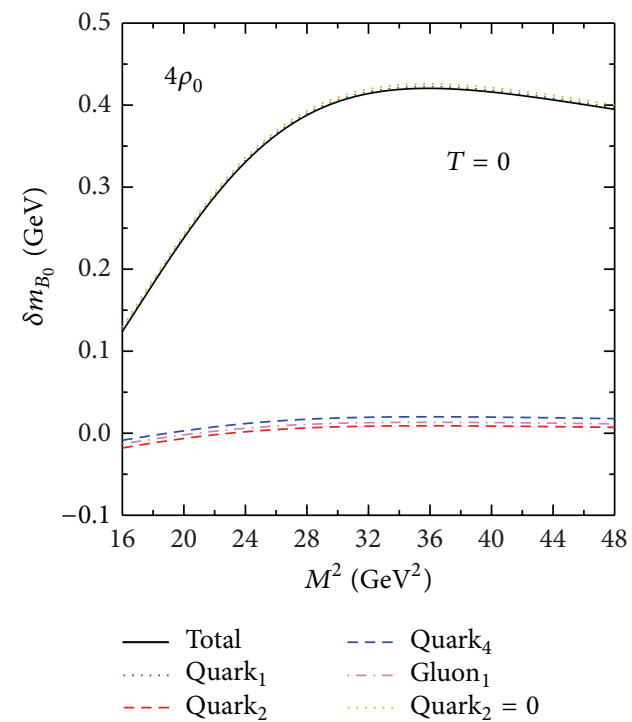

(e)

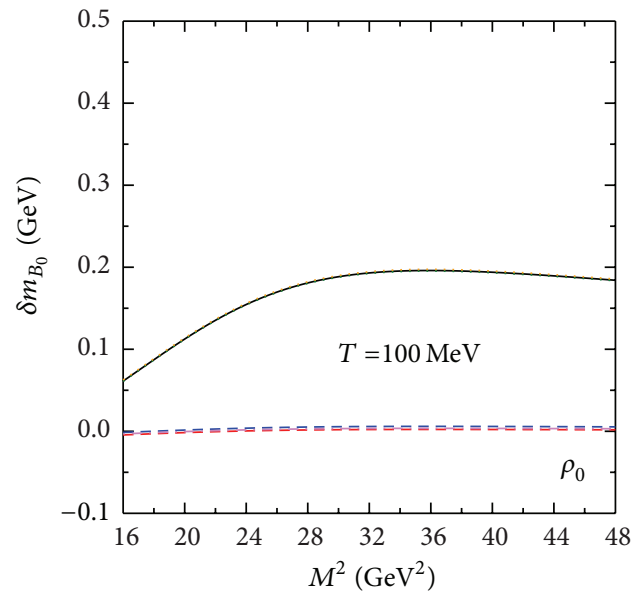

(b)

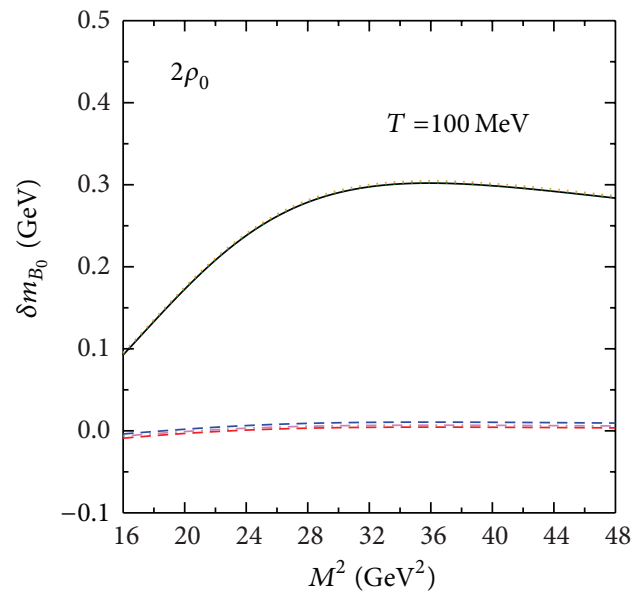

(d)

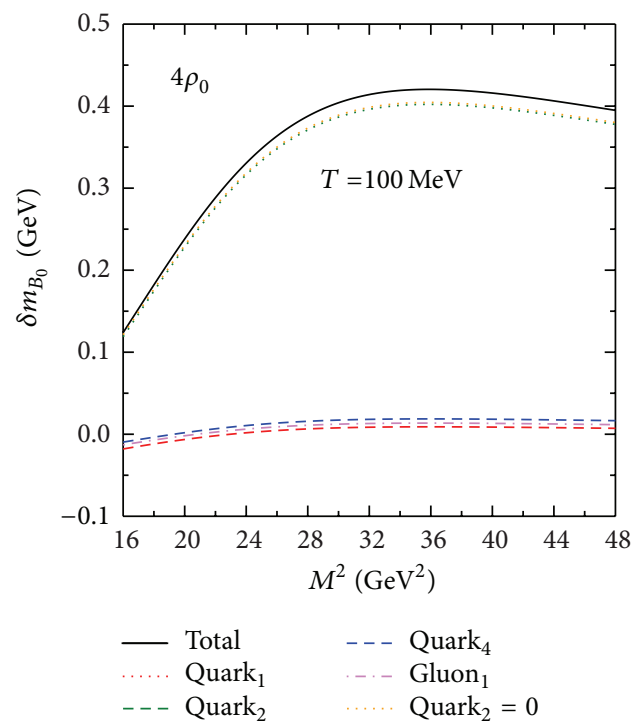

(f)

FIgURE 9: (Color online) Figure shows the contribution of individual condensates to the mass shift of scalar vector mesons $B_{0}$ as a function of the squared Borel mass parameter, $M^{2}$. The subplots (a), (c), and (e) show the results at temperature $T=0$, whereas the subplots (b), (d), and (f) are plotted for temperature $T=100 \mathrm{MeV}$. We have shown the results at baryon densities $\rho_{0}, 2 \rho_{0}$, and $4 \rho_{0}$. 


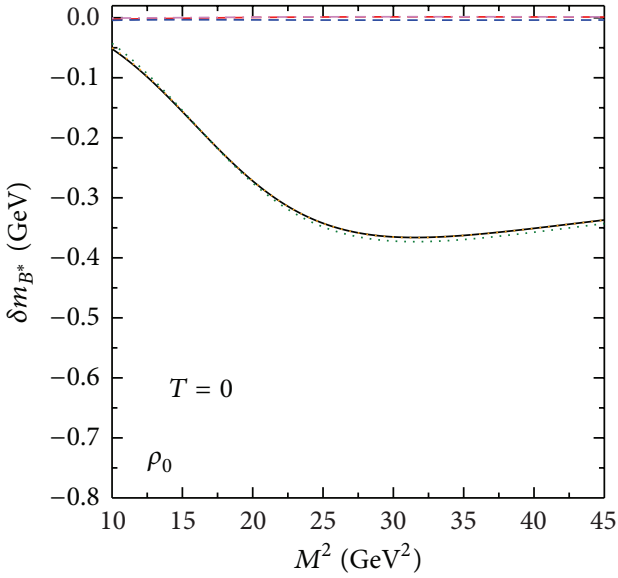

(a)

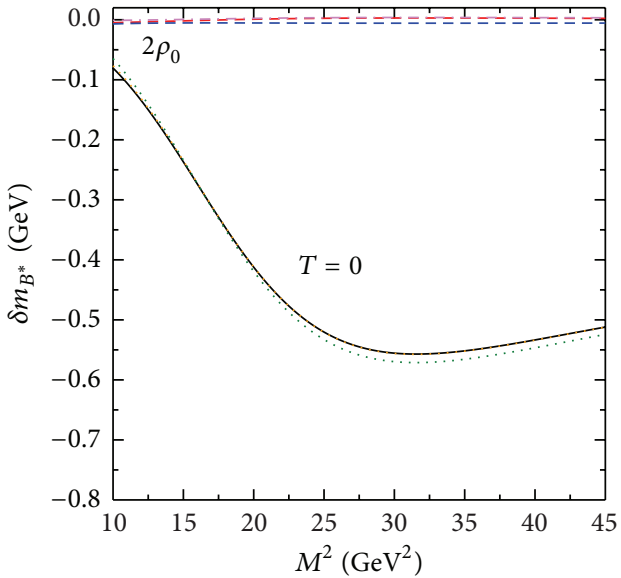

(c)

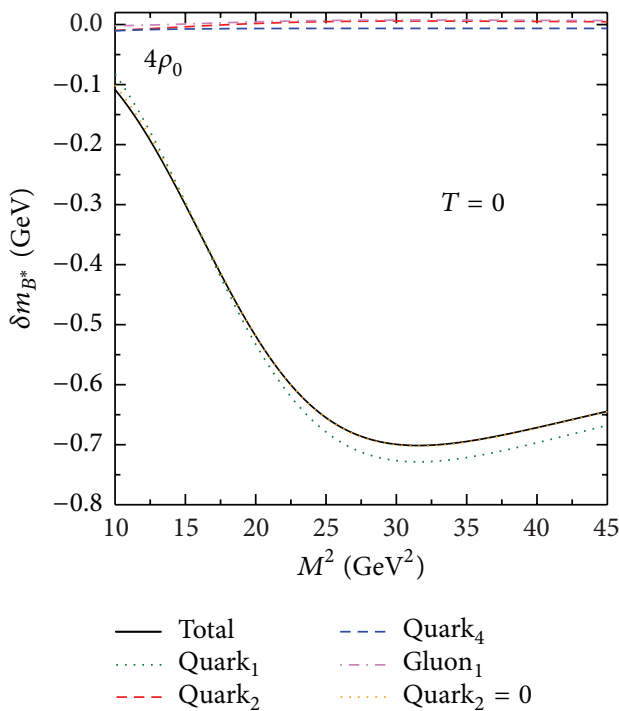

(e)

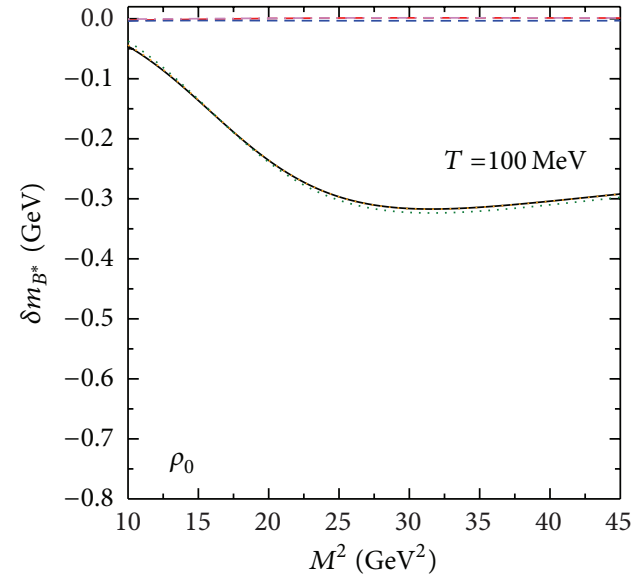

(b)

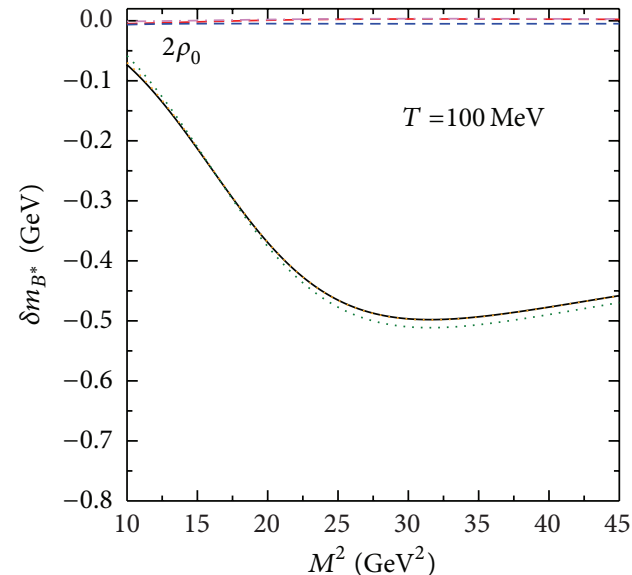

(d)

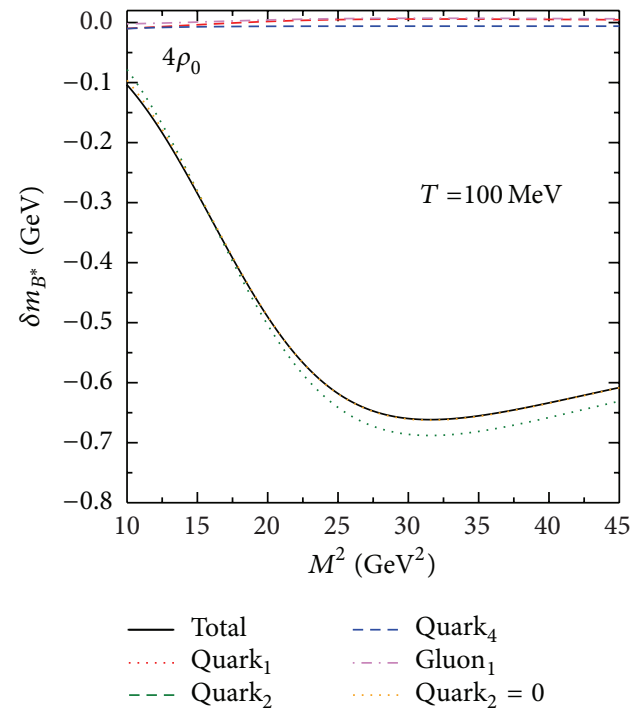

(f)

FIGURE 10: Figure shows the contribution of individual condensates to the mass shift of vector mesons $B^{*}$ as a function of the squared Borel mass parameter, $M^{2}$. The subplots (a), (c), and (e) show the results at temperature $T=0$, whereas the subplots (b), (d), and (f) are plotted for temperature $T=100 \mathrm{MeV}$. We have shown the results at baryon densities $\rho_{0}, 2 \rho_{0}$, and $4 \rho_{0}$. 


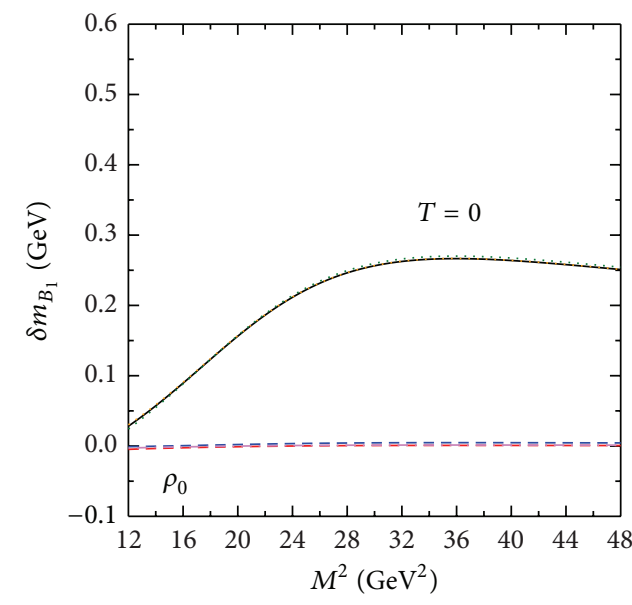

(a)

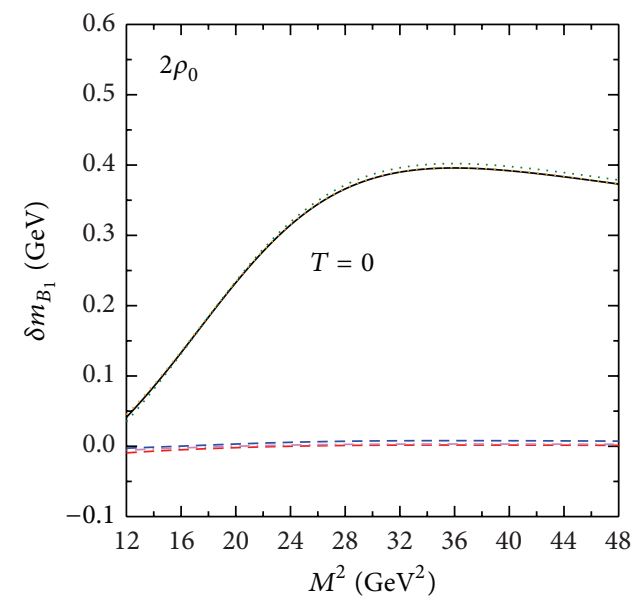

(c)

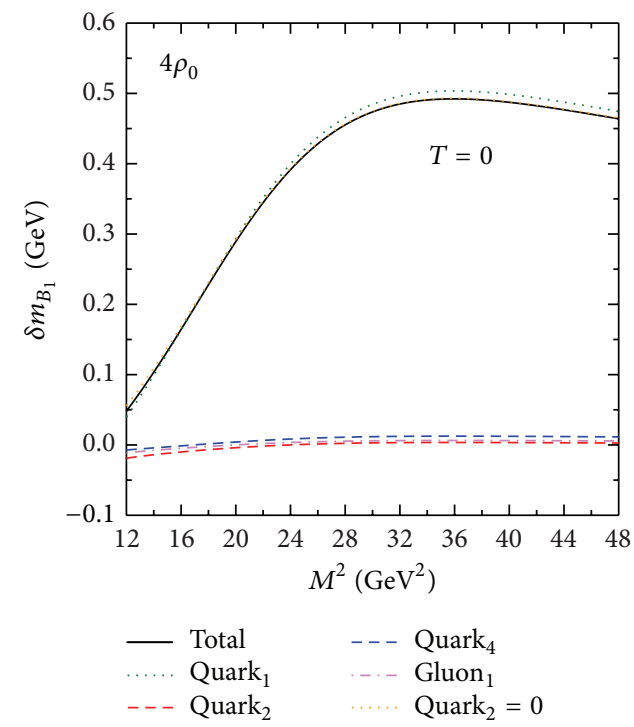

(e)

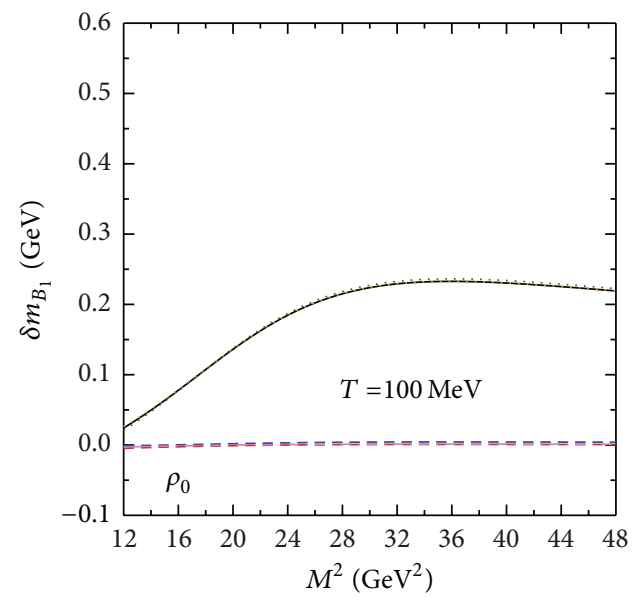

(b)

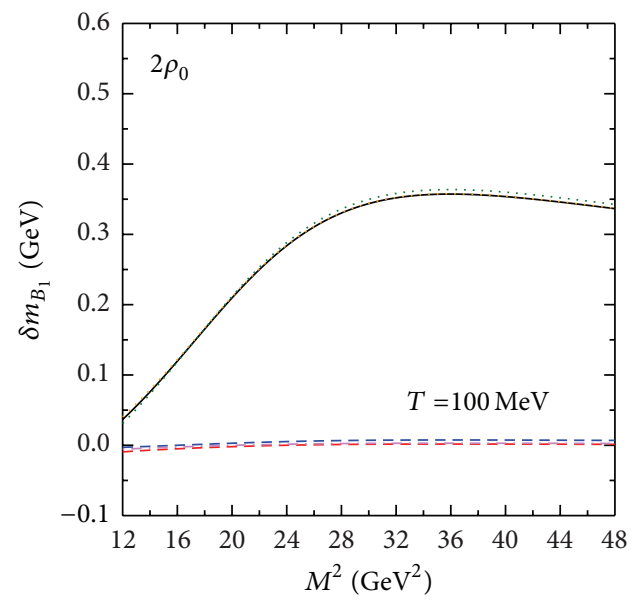

(d)

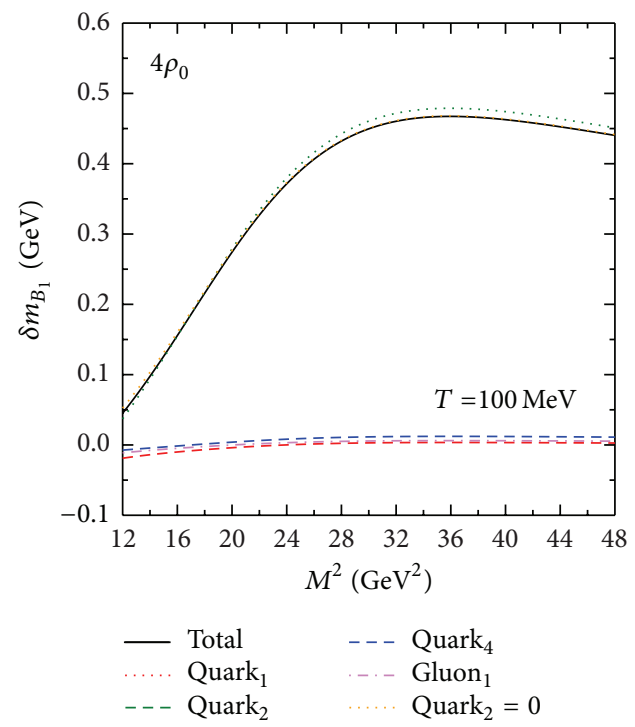

(f)

FIGURE 11: (Color online) Figure shows the contribution of individual condensates to the mass shift of axial-vector mesons $B_{1}$ as a function of the squared Borel mass parameter, $M^{2}$. The subplots (a), (c), and (e) show the results at temperature $T=0$, whereas the subplots (b), (d), and (f) are plotted for temperature $T=100 \mathrm{MeV}$. We have shown the results at baryon densities $\rho_{0}, 2 \rho_{0}$, and $4 \rho_{0}$. 
collisions. However, for the vector mesons $D^{*}$ we observe the negative values of mass shift and hence we conclude that they feel attractive interactions in the nuclear medium. The decrease in the mass of vector mesons $D^{*}$ may cause the decay of higher charmonium states to $D^{*} \bar{D}^{*}$ pairs and hence it may be a cause of $J / \psi$ suppression in heavy-ion collision experiments.

\section{Summary}

In the present paper we investigated the mass modifications of scalar mesons $\left(D_{0}, B_{0}\right)$, vector mesons $\left(D^{*}, B^{*}\right)$ and axialvector mesons $\left(D_{1}, B_{1}\right)$ at finite density and temperature of the nuclear medium. We used QCD sum rules along with chiral SU(3) model to investigate the properties of above mentioned mesons. Using chiral SU(3) model we found the values of quark and gluon condensates at finite density and temperature of the medium which were further used within QCD sum rules to find the in-medium masses of scalar, vector, and axial-vector mesons. We observed a positive value of mass shift for the scalar mesons $\left(D_{0}\right.$ and $\left.B_{0}\right)$ and axialvector mesons $\left(D_{1}\right.$ and $\left.B_{1}\right)$; that is, their in-medium mass was found to be more than the vacuum mass. For a constant value of temperature the values of mass shift for these scalar and axial-vector mesons are found to increase as we increase the density of the nuclear medium. On the other hand for a constant value of density the temperature of the medium causes a decrease in the values of mass shift of scalar and axial-vector mesons. The vector mesons $D^{*}$ and $B^{*}$ are found to have negative values of mass shift. It means their inmedium masses are small as compared to vacuum masses. For a constant value of density, as a function of temperature, the magnitude of mass shift of $D^{*}$ and $B^{*}$ mesons decreases. However, for a constant value of temperature, as a function of density the magnitude of mass shift of vector mesons $D^{*}$ and $B^{*}$ are found to increase. We have also investigated the effects of individual terms on the mass shift of mesons. It was found that the scalar quark condensates, $\bar{q} q$, have maximum contribution to the in-medium modification of scalar, vector, and axial-vector mesons. The effects of density and temperature of the medium on the scattering lengths of scalar, vector, and axial-vector mesons were also investigated. We observed the positive value of scattering lengths for scalar and axial-vector mesons, whereas for the vector mesons a negative value of scattering lengths were observed. Also it was found that the magnitude of the scattering lengths of scalar, vector, and axial-vector mesons decreases as we move from low to high value of density or temperature of the nuclear medium. The present investigation of medium modification of scalar, vector, and axial-vector mesons will be helpful for understanding their production rate and also the phenomenon of $J / \psi$ suppression in the compressed baryonic matter experiment at FAIR, GSI.

\section{Conflict of Interests}

The author declares that there is no conflict of interests regarding the publication of this paper.

\section{Acknowledgments}

The author is thankful to Amruta Mishra for fruitful discussions on chiral SU(3) model and QCD sum rules. The financial support from the Department of Science and Technology (DST), India, for research project under fast track scheme for young scientists (SR/FTP/PS-209/2012) is gratefully acknowledged.

\section{References}

[1] W. S. Chung, C. M. Ko, and G. Q. Li, "Seeing phi mesons through dileptons," Nuclear Physics A, vol. 641, no. 3, pp. 357378, 1998.

[2] D. B. Kaplan and A. E. Nelson, "Strange goings on in dense nucleonic matter," Physics Letters B, vol. 175, no. 1, pp. 57-63, 1986.

[3] A. E. Nelson and D. B. Kaplan, "Strange condensate realignment in relativistic heavy ion collisions," Physics Letters B, vol. 192, no. 1-2, pp. 193-197, 1987.

[4] G. E. Brown and M. Rho, "Chiral restoration in hot and/or dense matter," Physics Reports, vol. 269, no. 6, pp. 333-380, 1996.

[5] P. Papazoglou, D. Zschiesche, S. Schramm, J. Schaffner-Bielich, H. Stöcker, and W. Greiner, "Nuclei in a chiral SU(3) model," Physical Review C-Nuclear Physics, vol. 59, no. 1, pp. 411-427, 1999.

[6] A. Mishra, E. L. Bratkovskaya, J. Schaffner-Bielich, S. Schramm, and H. Stöcker, "Kaons and antikaons in hot and dense hadronic matter," Physical Review C, vol. 70, no. 4, Article ID 044904, 2004.

[7] A. Mishra and S. Schramm, "Isospin dependent kaon and antikaon optical potentials in dense hadronic matter," Physical Review C, vol. 74, Article ID 064904, 2006.

[8] A. Mishra, S. Schramm, and W. Greiner, "Kaons and antikaons in asymmetric nuclear matter," Physical Review C, vol. 78, Article ID 024901, 2008.

[9] A. Mishra, A. Kumar, S. Sanyal, and S. Schramm, "Kaon and antikaon optical potentials in isospin asymmetric hyperonic matter," The European Physical Journal A, vol. 41, no. 2, pp. 205213, 2009.

[10] A. Mishra and A. Mazumdar, "D mesons in asymmetric nuclear matter," Physical Review C, vol. 79, Article ID 024908, 2009.

[11] A. Mishra, E. L. Bratkovskaya, J. Schaffner-Bielich, S. Schramm, and H. Stöcker, "Mass modification of D meson in hot hadronic matter," Physical Review C, vol. 69, Article ID 015202, 2004.

[12] A. Kumar and A. Mishra, " $D$ mesons and charmonium states in asymmetric nuclear matter at finite temperatures," Physical Review C, vol. 81, Article ID 065204, 2010.

[13] A. Kumar and A. Mishra, "D-mesons and charmonium states in hot isospin asymmetric strange hadronic matter," The European Physical Journal A, vol. 47, article 164, 2011.

[14] S. H. Lee and K. Morita, "Charmonium states in quark-gluon plasma," Pramana, vol. 72, no. 1, pp. 97-108, 2009.

[15] K. Morita and S. H. Lee, "Critical behavior of charmonia across the phase transition: a QCD sum rule approach," Physical Review C, vol. 77, Article ID 064904, 2008.

[16] F. Klingl, S. Kim, L. Su Houng et al., "Masses of J/ $\psi$ and $\eta c$ in the nuclear medium: QCD sum rule approach," Physical Review Letters, vol. 82, p. 3396, 1999.

[17] Y. Koike and A. Hayashigaki, "QCD sum rules for $\rho, \omega, \varphi$ meson-nucleon scattering lengths and the mass shifts in nuclear 
medium," Progress of Theoretical Physics, vol. 98, no. 3, pp. 631652, 1997.

[18] T. Hilger, R. Thomas, and B. Kämpfer, "QCD sum rules for $D$ and $B$ mesons in nuclear matter," Physical Review C, vol. 79, no. 2, Article ID 025202, 2009.

[19] A. Hayashigaki, "Mass modification of $D$-meson at finite density in QCD sum rule," Physics Letters B, vol. 487, no. 1-2, pp. 96-103, 2000.

[20] E. Oset and A. Ramos, "Non-perturbative chiral approach to swave KN̄ interactions," Nuclear Physics A, vol. 635, no. 1-2, pp. 99-120, 1998.

[21] V. Koch, " $K{ }^{-}$-proton scattering and the $\Lambda$ (1405) in dense matter," Physics Letters B, vol. 337, no. 1-2, pp. 7-13, 1994.

[22] L. Tolós, D. Cabrera, and A. Ramos, "Strange mesons in nuclear matter at finite temperature," Physical Review C, vol. 78, Article ID 045205, 2008.

[23] Z.-G. Wang and T. Huang, "In-medium mass modifications of the $D_{0}$ and $\mathrm{B}_{0}$ mesons from QCD sum rules," Physical Review $C$, vol. 84, Article ID 048201, 2011.

[24] Z. Wang, "Analysis of mass modifications of the vector and axial-vector heavy mesons in the nuclear matter with the QCD sum rules," International Journal of Modern Physics A, vol. 28, Article ID 1350049, 2013.

[25] L. Tolos, R. Molina, D. Gamermann, and E. Oset, "Charm and hidden charm scalar resonances in nuclear matter," Nuclear Physics A, vol. 827, no. 1-4, pp. 249c-251c, 2009.

[26] T. Hilger and B. Kampfer, "In-medium modifications of scalar charm mesons in nuclear matter," Nuclear Physics BProceedings Supplements, vol. 207, pp. 277-280, 2010.

[27] N. Masera for the HELIOS-3 Collaboration, "Dimuon production below mass $3.1-\mathrm{GeV} / \mathrm{c} * * 2$ in $\mathrm{p} \mathrm{W}$ and $\mathrm{S} \mathrm{W}$ interactions at 200-A/GeV/c," Nuclear Physics A, vol. 590, pp. 93C-102C, 1995.

[28] G. Agakichiev et al., "Enhanced production of low-mass electron pairs in $200 \mathrm{GeV} /$ nucleon S-Au collisions at the CERN super proton synchrotron," Physical Review Letters, vol. 75, Article ID 1272, 1995.

[29] G. Agakichiev, R. Baur, P. Braun-Munzinger et al., "Low-mass $\mathrm{e}^{+} \mathrm{e}^{-}$pair production in $158 \mathrm{~A} \mathrm{GeV} \mathrm{Pb-Au} \mathrm{collisions} \mathrm{at} \mathrm{the} \mathrm{CERN}$ SPS, its dependence on multiplicity and transverse momentum," Physics Letters B, vol. 422, no. 1-4, pp. 405-412, 1998.

[30] B. Lenkeit, G. Agakichiev, H. Appelshäuser et al., "Recent results from $\mathrm{Pb}-\mathrm{Au}$ collisions at $158 \mathrm{GeV} / \mathrm{c}$ per nucleon obtained with the CERES spectrometer," Nuclear Physics A, vol. 661, pp. 23-32, 1999.

[31] R. J. Porter et al., "Dielectron cross section measurements in nucleus-nucleus reactions at $1.0 \mathrm{~A} \mathrm{GeV,"} \mathrm{Physical} \mathrm{Review} \mathrm{Letters,}$ vol. 79, p. 1229, 1997.

[32] W. K. Wilson, S. Beedoe, R. Bossingham (The DLS Collaboration) et al., "Inclusive dielectron cross sections in $\mathbf{p}+\mathbf{p}$ and $\mathbf{p}+\mathbf{d}$ interactions at beam energies from 1.04 to $4.88 \mathrm{GeV}$,' Physical Review C, vol. 57, article 1865, 1998.

[33] A. Andronic, P. Braun-Munzinger, K. Redlich, and J. Stachel, "Charmonium and open charm production in nuclear collisions at SPS/FAIR energies and the possible influence of a hot hadronic medium," Physics Letters, Section B: Nuclear, Elementary Particle and High-Energy Physics, vol. 659, no. 1-2, pp. 149-155, 2008.

[34] A. Kumar and A. Mishra, "J/ $\psi$ and $\eta c$ masses in isospin asymmetric hot nuclear matter: a QCD sum rule approach," Physical Review C, vol. 82, Article ID 045207, 2010.
[35] D. Zschiesche, A. Mishra, S. Schramm, H. Stöcker, and W. Greiner, "In-medium vector meson masses in a chiral SU(3) model," Physical Review C, vol. 70, Article ID 045202, 2004.

[36] A. Mishra, K. Balazs, D. Zschiesche, S. Schramm, H. Stöcker, and W. Greiner, "Effects of Dirac sea polarization on hadronic properties: a chiral SU(3) approach," Physical Review C, vol. 69, Article ID 024903, 2004.

[37] S. Weinberg, "Nonlinear realizations of chiral symmetry," Physical Review, vol. 166, no. 5, pp. 1568-1577, 1968.

[38] S. Coleman, J. Wess, and B. Zumino, "Structure of phenomenological lagrangians. I," Physical Review, vol. 177, no. 5, pp. 22392247, 1969.

[39] C. G. Callan, S. Coleman, J. Wess, and B. Zumino, "Structure of phenomenological lagrangians. II," Physical Review, vol. 177, no. 5, pp. 2247-2250, 1969.

[40] W. A. Bardeen and B. W. Lee, "Some considerations on nonlinear realizations of chiral SU(3) $\times \mathrm{SU}(3)$," Physical Review Letters, vol. 177, article 2389, 1969.

[41] J. Schechter, "Effective Lagrangian with two color-singlet gluon fields," Physical Review D, vol. 21, Article ID 3393, 1980.

[42] Y. Kwon, C. Sasaki, and W. Weise, "Vector mesons at finite temperature and QCD sum rules," Physical Review C-Nuclear Physics, vol. 81, no. 6, Article ID 065203, 2010.

[43] S. Zschocke, O. P. Pavlenko, and B. Kampfer, "Evaluation of QCD sum rules for light vector mesons at finite density and temperature," The European Physical Journal A, vol. 15, pp. 529$537,2002$.

[44] R. Thomas, T. Hilger, and B. Kampfer, "Four-quark condensates in nucleon QCD sum rules," Nuclear Physics A, vol. 795, no. 1-4, pp. 19-46, 2007.

[45] R. J. Furnstahl and B. D. Serot, "Covariant mean-field calculations of finite-temperature nuclear matter," Physical Review C, vol. 41, no. 1, pp. 262-279, 1990.

[46] J. Theis, G. Graebner, G. Buchwald, J. Maruhn, W. Greiner, and J. Polonyi, "Phase transition of the nucleon-antinucleon plasma in a relativistic mean-field theory," Physical Review D, vol. 28, p. 2286, 1983.

[47] J. Sollfrank and U. Heinz, "Chiral and gluon condensates at finite temperature," Zeitschrift für Physik C Particles and Fields, vol. 65, no. 1, pp. 111-121, 1995.

[48] Z. Li-Juan, Z. Bo, Z. Hong-Wei, and M. Wei-Xing, "Temperature dependence of Quark and Gluon condensate in the Dyson-Schwinger equations at finite temperature," http://arxiv-web3.library.cornell.edu/abs/1403.6965.

[49] D. E. Miller, "The gluon condensate in QCD at finite temperature," Acta Physica Polonica B, vol. 28, p. 2937, 1997.

[50] Y.-H. Tan, X.-B. Zhang, L. Li, and P.-Z. Ning, "K ${ }^{+}$scattering with the nuclear pion from chiral effective Lagrangian," Communications in Theoretical Physics, vol. 37, pp. 215-220, 2002.

[51] M. Lutz, "Nuclear kaon dynamics," Physics Letters B, vol. 426, no. 1-2, pp. 12-20, 1998.

[52] A. Ramos and E. Oset, "The properties of $\bar{K}$ in the nuclear medium," Nuclear Physics A, vol. 671, pp. 481-502, 2000.

[53] M. F. M. Lutz and C. L. Korpa, "Self-consistent propagation of hyperons and antikaons in nuclear matter based on relativistic chiral SU(3) dynamics," Nuclear Physics A, vol. 700, no. 1-2, pp. 309-329, 2002.

[54] L. Tolós, A. Ramos, and A. Polls, "Antikaon nuclear potential in hot and dense matter," Physical Review C, vol. 65, Article ID 054907, 2002. 

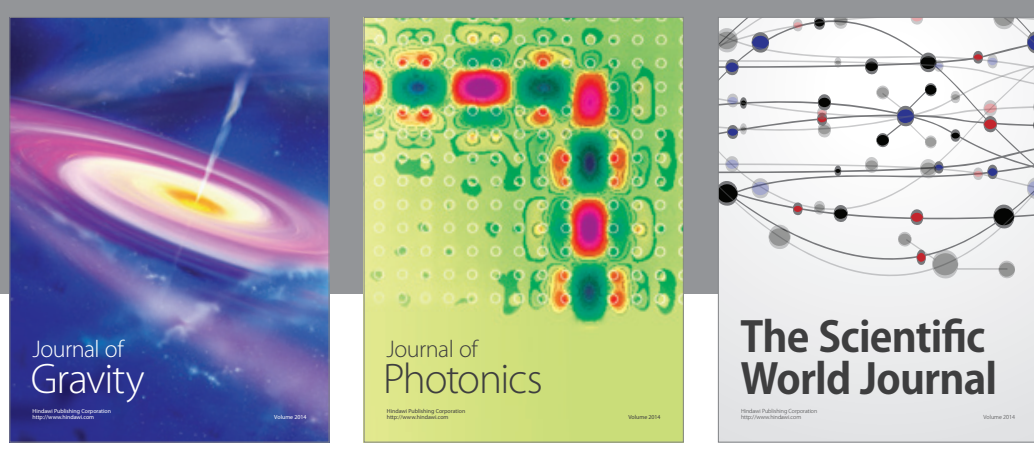

The Scientific World Journal
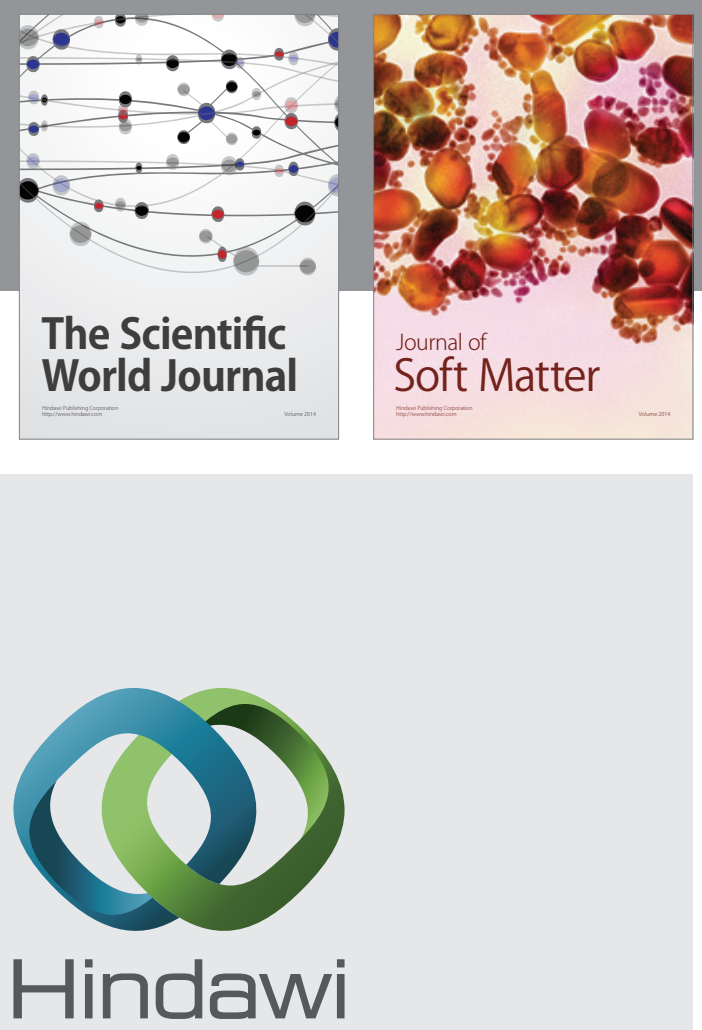

Submit your manuscripts at

http://www.hindawi.com

nternational Journal of

Statistical Mechanics
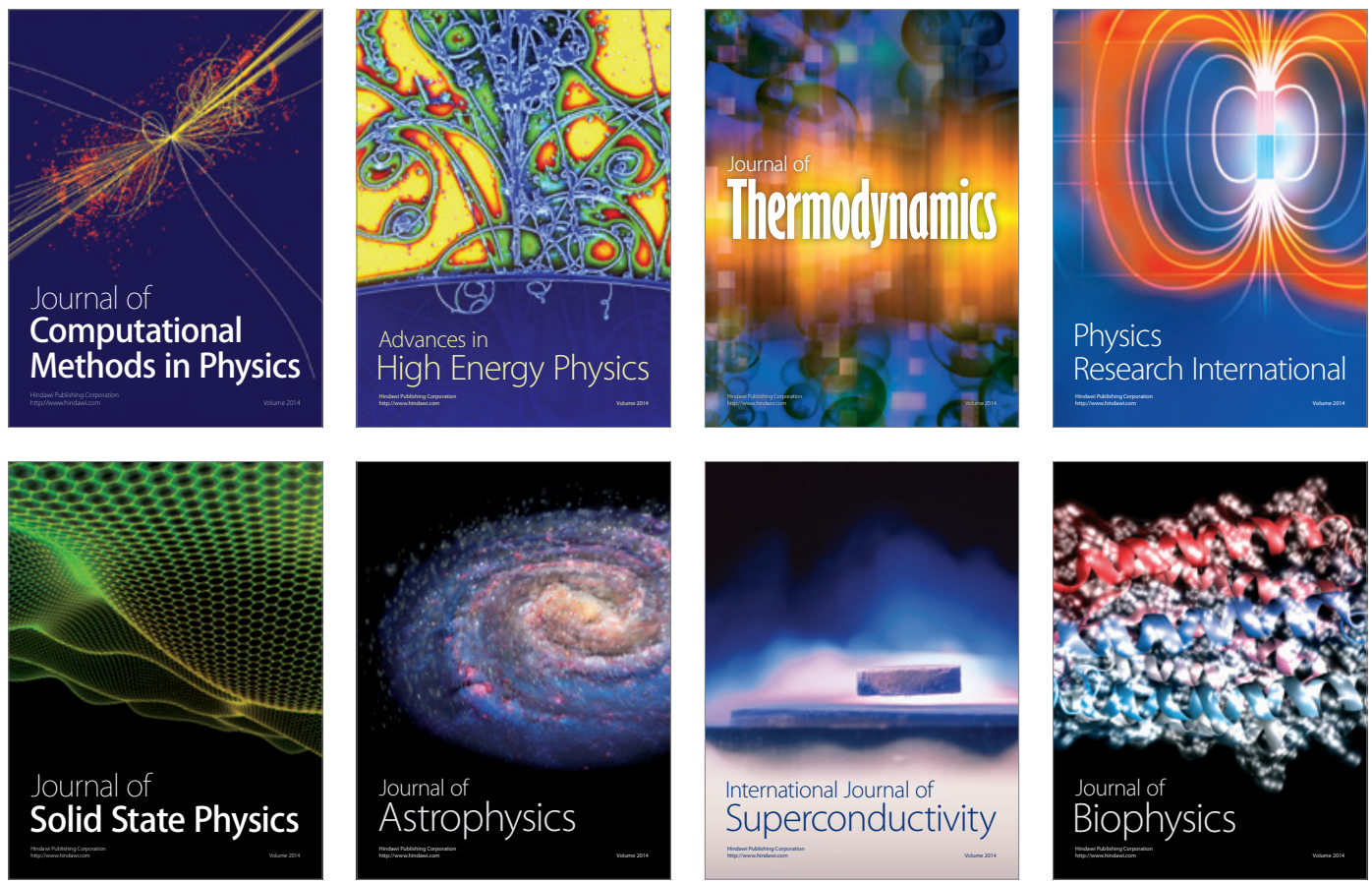
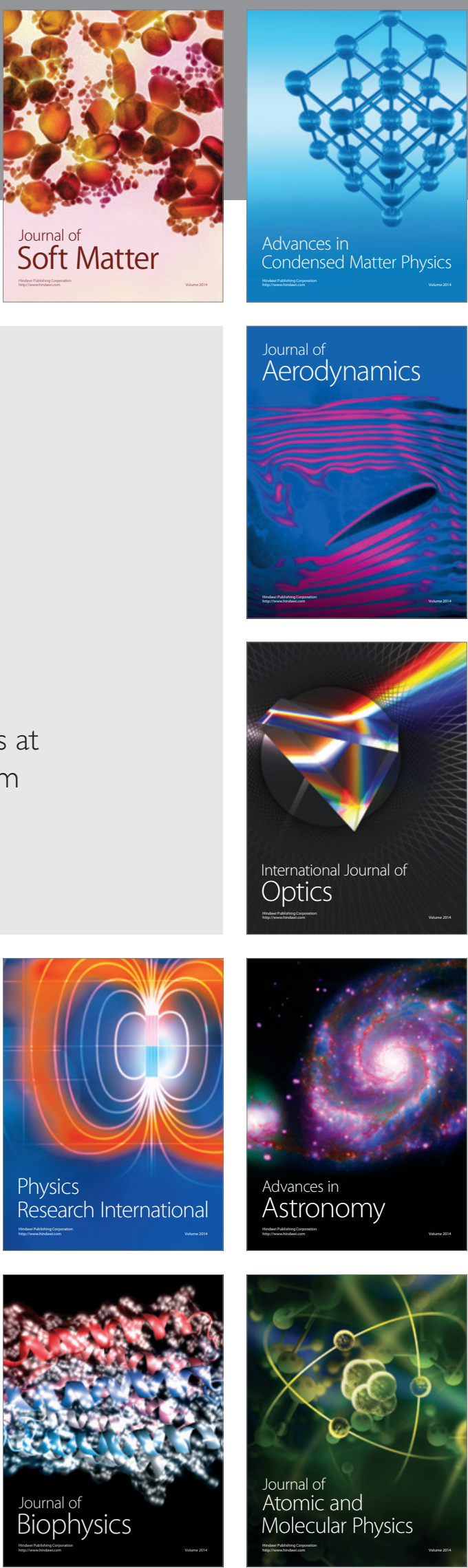